\title{
Engineered Heart Tissue Models from hiPSC-Derived Cardiomyocytes and Cardiac ECM for Disease Modeling and Drug Testing Applications
}

${ }^{1,2}$ Idit Goldfracht, ${ }^{3}$ Yael Efraim, ${ }^{1}$ Rami Shinnawi, ${ }^{1}$ Ekaterina Kovalev, ${ }^{1}$ Irit Huber, ${ }^{1}$ Amira Gepstein, ${ }^{1}$ Gil Arbel, ${ }^{1}$ Naim Shaheen, ${ }^{3,4}$ Malte Tiburcy, ${ }^{3,4}$ Wolfram H Zimmerman, ${ }^{5}$ Marcelle Machluf, and ${ }^{1,6}$ Lior Gepstein.

${ }^{1}$ Sohnis Research Laboratory for Cardiac Electrophysiology and Regenerative Medicine; the Rappaport Faculty of Medicine and Research Institute, Technion - Israel Institute of Technology

${ }^{2}$ Interdisciplinarry Biotechnology Program. Technion - Israel Institute of Technology

${ }^{3}$ Institute of Pharmacology and Toxicology, University Medical Center Goettingen, Germany

${ }^{4}$ DZHK (German Center for Cardiovascular Research), partner site Goettingen, Germany

${ }^{5}$ Faculty of Biotechnology and Food Engineering, Technion - Israel Institute of Technology

${ }^{6}$ Cardiology Department, Rambam Health Care Campus.

Correspondence: Lior Gepstein, MD, PhD. Rappaport Faculty of Medicine and Research Institute, Technion - Israel Institute of technology. POB 9649, Haifa, 31096, Israel.

E-mail: mdlior@ @echnion.ac.il, Tel: 972-4-7772180 


\begin{abstract}
Cardiac tissue engineering provides unique opportunities for cardiovascular disease modeling, drug testing, and regenerative medicine applications. To recapitulate human heart tissue, we combined human induced pluripotent stem cell-derived cardiomyocytes (hiPSC-CMs) with a chitosan-enhanced extracellular-matrix (ECM) hydrogel, derived from decellularized pig hearts. Ultrastructural characterization of the ECM-derived engineered heart tissues (ECM-EHTs) revealed an anisotropic muscle structure, with embedded cardiomyocytes showing more mature properties than 2D-cultured hiPSC-CMs. Force measurements confirmed typical force-length relationships, sensitivity to extracellular calcium, and adequate ionotropic responses to contractility modulators. By combining genetically-encoded calcium and voltage indicators with laser-confocal microscopy and optical mapping, the electrophysiological and calcium-handling properties of the ECM-EHTs could be studied at the cellular and tissue resolutions. This allowed to detect drug-induced changes in contraction rate (isoproterenol, carbamylcholine), optical signal morphology (E-4031, ATX2, isoproterenol, ouabin and quinidine), cellular arrhythmogenicity (E-4031 and ouabin) and alterations in tissue conduction properties (lidocaine, carbenoxolone and quinidine). Similar assays in ECM-EHTs derived from patient-specific hiPSC-CMs recapitulated the abnormal phenotype of the long QT syndrome and catecholaminergic polymorphic ventricular tachycardia. Finally, programmed electrical stimulation and drug-induced pro-arrhythmia led to the development of reentrant arrhythmias in the ECM-EHTs. In conclusion, a novel ECM-EHT model was established, which can be subjected to high-resolution long-term serial functional phenotyping, with important implications for cardiac disease modeling, drug testing and precision medicine.
\end{abstract}

Key words: Cardiac tissue engineering, induced pluripotent stem cells, cardiac extracellular matrix, optical mapping, genetically encoded voltage/calcium reporters, arrhythmogenic syndromes 


\section{Introduction}

The ability to reprogram adult somatic cells into human induced pluripotent stem cells (hiPSC) [1], which can then be differentiated into patient-specific hiPSC-derived cardiomyocytes (hiPSC-CMs) [2, 3], provided unique cellular tools for the fields of cardiac regenerative medicine [4-6], disease modeling [7-9], drug testing [10-13] and precision medicine [11, 14, 15]. Yet, not every aspect of the human myocardium can be studied at the single cell level. For example, the tissue's mechanical force generation, electrical conduction properties and reentrant arrhythmias are processes that can only be studied at the tissue levels. These shortcomings could be addressed by the emerging field of cardiac tissue engineering $[16,17]$, which aims to generate heart tissue surrogates by combining cells (including hiPSC-CMs) and scaffolding polymers. The resulting engineered tissues can be utilized for myocardial tissue replacement [18-20], for physiological studies, for studying multicellular interactions, and for several drug testing and disease modeling applications [21-29].

The three-dimensional environment, multicellular interactions, extracellular matrix, and applied mechanical and electrical stimuli involved in cardiac tissue engineering were all shown to enhance hiPSC-CMs maturation to a greater extent than in traditional two-dimensional cell/tissue culturing [21, 26-31]. Different types of scaffolds have been used in cardiac tissue engineering, including those derived from synthetic polymers [31] and from natural biological materials such as collagen [32] and fibrin [33]).

The native cardiac extracellular matrix (ECM) provides a unique micro-environment for resident cardiomyocytes that supports the cells in multiple ways [34]. It is not surprising therefore that the use of decellularized hearts as scaffolds for cardiac tissue engineering approaches has become an attractive option [35-39]. One of the limitations in using such approaches, however, is that the shape and size of the derived patches are limited by the original tissue structure. To address this shortcoming, new protocols were recently developed that allow to dissolve and modify the decellularized heart ECM. 
This led to the development of a solubilized porcine cardiac ECM (pcECM) hydrogel that can be solidified in any desirable shape $[40,41]$. In this study, we aimed to derive ring-shaped engineered heart tissues (EHTs) by combining aforementioned pcECM gel, strengthened by adding $0.2 \%$ chitosan and 0.01gr genipin/gr ECM (for cross-linking), with different types of hiPSC-CMs. We chose to use this specific pcECM gel formulation since in our previous study [40] it displayed mechanical properties that were similar to that of the adult heart [42-45].

The hiPSC-CMs used for creation of the ECM-EHTs were derived from healthy individuals or from patients with the inherited arrhythmogenic disorders, long QT syndrome (LQTS) [9] and catecholaminergic polymorphic ventricular tachycardia (CPVT) [14]. We next evaluated the potential role of the established healthy and patient/disease-specific ECM-EHTs to serve as suitable surrogate models for the human heart, focusing on studying their electrophysiological and mechanical properties.

To achieve this goal we utilized a variety of novel experimental tools including: (1) the use of genetically-encoded calcium and voltage indicators (GECIs and GEVIs) [13, 46] for evaluation of the tissues' action-potential (AP) and calcium-handling properties; (2) optical mapping strategies for assessment of conduction and arrhythmogenesis; (3) targeted pharmacology; and (4) mechanical force assessment for studying the tissues' contractile properties. Using these tissue characterization strategies we demonstrated that the established ECM-EHTs can serve as unique experimental models for various physiological, drug testing, and disease modeling application.

\section{Materials and Methods}

\subsection{Decellularized porcine heart and ECM gel preparation}

Porcine cardiac tissues, derived from 5-6 month old animals, were decellularized according to our previously published protocols with slight modifications [39]. Briefly, two cycles of alternating hyper/hypo tonic $\mathrm{NaCl}$ solutions, trypsin enzymatic reaction, and detergent washes with Triton-X-100 
were conducted to isolate the cardiac ECM. The ECM was then lyophilized and solubilized in $\mathrm{HCl}$ $(0.01 \mathrm{M})$ to a concentration of $10 \mathrm{mg} / \mathrm{ml}$ using sonication (1min) and pepsin enzymatic digestion $(1 \mathrm{mg} / \mathrm{ml})$ as previously described [40]. $\mathrm{NaOH}$ was then added to adjust the solution to $\mathrm{pH}=5$. The pcECM solution was mixed with chitosan, a natural biocompatible polysaccharide of molecular weight between 50,000 and 150,000 gr/mol and 75-90 degree of deacetylation (FMC Biopolymer, Norway, 0.2 gr chitosan/gr ECM), and also with the crosslinker genipin (0.01gr genipin/gr ECM), in order to control the resulting mechanical properties as described [40].

\subsection{Propagation of hiPSCs and cardiomyocyte differentiation}

A healthy-control [13] and two patient-specific $[9,14]$ hiPSC lines, previously created in our lab, were used in this study. The hiPSCs were generated by reprograming of dermal fibroblasts obtained from a healthy-control young man [13], from a young female patient diagnosed with the long QT syndrome type 2 (LQTS2) [9], and from a patient diagnosed with catecholaminergic polymorphic ventricular tachycardia type 2 (CPVT2) [14]. The fibroblasts were reprogrammed to generate the hiPSC by retroviral transduction of SOX2, KLF4 and OCT4 as previously described [6]. The establishment of these lines and all the studies performed were approved by the IRB committee (Helsinki committee) of Rambam Health Care Campus. In addition, we also utilized a previously established transgenic hiPSC line expressing the GEVI, ArcLight [13].

Colonies of hiPSCs were propagated on Matrigel using mTeSR-1 (stem-cell technologies). Cardiomyocyte differentiation was based on a previously described protocol [47]. Briefly, to induce differentiation, three to five days after passaging or when the cells reached $80-90 \%$ confluence (day 0 of differentiation), the culture-medium was switched to a differentiation medium CDM3 [RPMI-1640 (Roswell Park Memorial Institute), recombinant human serum albumin 500 $\mu \mathrm{g} / \mathrm{mL}$ (Oryzogen), $213 \mu \mathrm{g} / \mathrm{mL}, \mathrm{L}$-ascorbic acid 2-phosphate (Sigma-Aldrich), $1 \%$ penicillin/streptomycin (100 U/ml and $100 \mathrm{~g} / \mathrm{ml}$, respectively; Biological Industries)], supplemented with $6 \mu \mathrm{mol} / \mathrm{L} \mathrm{CHIR} 99021$ (Stemgent) for 
two days. At day 2, the medium was replaced with CDM3 medium supplemented with $2 \mu \mathrm{M}$ Wnt-C59 (Selleckchem) instead of CHIR99021. From d4 onward, cells were cultured with CDM3 medium alone. The resulting beating monolayers (14-20d) were enzymatically dissociated into small clusters or single cardiomyocytes using TrypLE express (Gibco). Flow cytometry was performed routinely to ensure $>85 \%$ cardiomyocyte purity by analyzing the percentage of cells that expressed cTNT.

\subsection{Flow cytometry}

For the intracellular staining of cardiac troponin T (cTnT, Invitrogen), dissociated cardiomyocytes were fixed with $4 \%$ paraformaldehyde, permeabilized in phosphate-buffered saline (PBS) with 5\% fetal calf serum (FCS) and 0.5\% Saponin, and then stained for cTnT (1:500). Cells were stained with secondary antibodies and evaluated with LSR FortessaII flow cytometer (BD Biosciences) and FlowJo software.

\subsection{Generation of the engineered heart tissues (EHTs)}

EHT constructs were generated by combining the ECM pre-gel solution, 2x Dulbecco's Modified Eagle Medium; DMEM (containing 40\% 5×DMEM, 40\% fetal bovine serum, 15\% H2O, $10 \mu 1 / \mathrm{ml}$ glutamine, and $20 \mu \mathrm{l} / \mathrm{ml}$ penicillin/streptomycin), $0.1 \mathrm{M} \mathrm{NaOH}$ and 2 million hiPSC-derived cardiomyocytes (hiPSC-CMs). The mixture was pipetted into circular casting molds [48] where the tissue condensed quickly. The resulting tissue was transferred onto passive-stretcher devices on $\mathrm{d} 3$. Medium was changed every other day (Iscove-Medium with $20 \%$ fetal bovine serum, $1 \%$ non-essential amino acids, $1 \%$ glutamine, $1 \%$ penicillin/streptomycin and $100 \mu \mathrm{mol} / 1 \beta$-mercaptoethanol).

\subsection{Immunostaining and electron microscopy}

EHTs were frozen and cryo-sectioned $(10 \mu \mathrm{m})$. The slides were permeabilized with $1 \%$ Triton X-100, blocked with 5\% horse serum, and incubated with primary antibodies targeting cardiac troponin I (cTnI, 1:150, Merck, MAB1691), collagen type I (1:150, Sigma-Aldrich, C2456) or ventricular myosin light chain-2 (MLC2v, 1:150, Abcam, ab 79935) at $4^{\circ} \mathrm{C}$ overnight. The specimens were washed and 
stained with appropriate secondary antibodies and DAPI. The preparations were examined using a Zeiss LSM-710 laser-scanning confocal microscope.

For transmission electron microscopy (TEM) analysis, samples were fixed in $3.5 \%$

glutaraldehyde and rinsed in $0.1 \mathrm{M}$ sodium cacodylate buffer, $\mathrm{pH}-7.4$. Tissue blocks $\left(1 \mathrm{~mm}^{3}\right)$ were postfixed with $2 \%$ OsO4 in $0.2 \mathrm{M}$ cacodylate buffer for $1 \mathrm{~h}$, rinsed again in cacodylate buffer to remove excess osmium, immersed in saturated aqueous uranyl acetate, dehydrated in graded alcohol solutions, immersed in propylene oxide, and embedded in Epon-812. Ultrathin sections (80nm) were mounted on 300-mesh, thin-bar copper grid, counterstained with saturated uranyl acetate and lead citrate. Sections were examined with a transmission electron microscope (Jeol-1011, JEM), at 80KV.

For scanning electron microscopy (SEM) analysis, EHTs were washed and submerged in distilled water, frozen and then lyophilized overnight. Samples were imaged using side- and back-scattered electrons (0.98Torr, 20kV), using a FEI-Quanta 200 electron microscope (OXFORD Instruments). Zeiss ULTRA plus field emission scanning electron microscope was used to image hiPSC-CMs within the EHTs. Samples were grown on $13 \mathrm{~mm}$ coverslips, fixed in paraformaldehyde and glutaraldehyde and then post fixed in osmium tetraoxide. They were further processed by dehydration through a graded ethanol series and finally coated in Iridium

\subsection{Real-time quantitative PCR}

Relevant hiPSCs-CMs and EHT-ECM were frozen in liquid-nitrogen. Total RNA was isolated using TRIzol (Invitrogen; 15596-026) according to the manufacturer's protocol. $1 \mu \mathrm{g}$ of total RNA was DNAse treated (DNAse I, Invitrogen) and reverse transcribed using superscript II reverse transcriptase (Invitrogen) with oligo-dT primers according to the manufacturer's protocol. RT-qPCR was performed using LightCycler 480 SYBR green master I (Roche) on a StepOnePlus Real-time PCR system (Applied Biosystems). Expression levels were normalized to the housekeeping gene GAPDH. Primer sequences are shown in Supplementary Table 1. 


\subsection{Lentiviral transduction of the ArcLight and GCaMP5G transgenes}

pLV-CAG-ArcLight and pLV-TroponinT-GCaMP5 plasmids were kindly provided by David Milan (Massachusetts General Hospital) and John Epstein (University of Pennsylvania), respectively. pLVGCaMP5G-ArchD95N was obtained from Addgene (plasmid 42168). HEK293T (Human embryonic kidney 293 cells) cells were transfected with $4.5 \mathrm{mg}$ of the relevant plasmid, $3 \mathrm{mg}$ of $\Delta \mathrm{NRF}$ packaging plasmid, and 1.5mg of the VSVG (vesicular stomatitis virus G) plasmid using jetPEI reagent (Polyplus). Fresh virus-containing media were collected at 48 and $72 \mathrm{hr}$ and used for two rounds of infections of the dissociated hiPSC-CMs to improve transduction efficiency. Viral dosages for the ArcLight and GCaMP5 plasmids were $7 \times 10^{5} \mathrm{TU} / \mathrm{ml}$ and $1.3 \times 10^{6} \mathrm{TU} / \mathrm{ml}$, respectively, resulting in multiplicity of infection (MOI) values for ArcLight and GCaMP5 transduction of 0.35 and 0.65 respectively. Transduction efficiency ranged between 20 to $30 \%$ in the different experiments. This was sufficient to allow robust functional $\mathrm{Ca}^{2+}$ and voltage imaging after creation of the EHTs in all cases.

\subsection{Laser-confocal AP and calcium transient recordings}

The line-scan mode of the Zeiss LSM710 laser-scanning confocal microscope was used to measure the fluorescence intensity of ArcLight and GCaMP5G expressing cells within the EHTs. This allowed the acquisition of optical APs and intracellular $\left[\mathrm{Ca}^{2+}\right]_{\mathrm{i}}$ transients at high-resolution, providing electrophysiological information at the cellular resolution (by sampling information from single-cells within the EHTs). The tissue was kept in a heated organ bath in Tyrode's solution (NaCl-140mM, KCl5.4mM, CaCl2-1.8mM, MgCl2-1mM, HEPES-10mM and glucose- $10 \mathrm{mM}$ ), and field stimulated using Warner-instruments stimulator. In addition, optical signals were also acquired at low-resolution; thus averaging data regarding the optical APs and intracellular $\left[\mathrm{Ca}^{2+}\right]_{i}$ transients from the entire tissue.

\subsection{Optical mapping}

To study conduction, ECM-EHTs were incubated in medium containing Blebbistatin $(5 \mu \mathrm{mol} / \mathrm{L}$, SigmaAldrich) for 30min to prevent motion artifacts. EHTs were further incubated with $25 \mu \mathrm{M}$ of the voltage- 
sensitive dye Di-4-ANBDQBS (purchased from Prof. Leslie Loew) for 10min at room temperature. Tissues were then excited using LEDs (X-Cite® TURBO, Excelitas Technologies) with a peak wavelength at $630 \mathrm{~nm}$. Imaging was performed at 260 frames/sec using a fast Electron Multiplying Charge-Coupled Device (EM-CCD) camera (Evolve® 512Delta, Photometrics, 512x512 pixels), mounted on an inverted macroscope (Olympus MVX10). The constructs were electrically pointstimulated using a bipolar electrode made of platinum/iridium (Alpha-Omega), which was mounted on a micromanipulator and connected to a stimulus isolation unit (SIU-102, Warner-instruments).

Induction of arrhythmias was achieved by rapid stimulation (sequential increase in pacing frequency until development of arrhythmias or loss of capture). Activation maps were generated by determining the local activation time (LAT) at each pixel as the time-point of the maximal first derivative (dF/dt) max of the fluorescent signal. A custom-designed software kindly provided by Prof. Bum-Rak Choi (Brown University) was used for creation of activation maps and analysis of conduction velocities (CVs).

\subsection{Pharmacological studies}

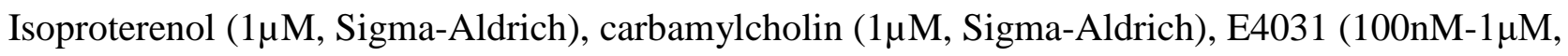
Alomone labs), ATX-II (30nM, Alomone labs), ouabain (1mM, Sigma-Aldrich) and quinidine (0.1, $0.3,1,3,10$, and $30 \mu \mathrm{M}$, Sigma-Aldrich) were dissolved in $\mathrm{H} 2 \mathrm{O}$, while dofetilide (25nM, SigmaAldrich) was dissolved in DMSO (Dimethyl sulfoxide). $\mathrm{Ca}^{2+}$ and AP recordings were performed $15 \mathrm{~min}$ after adding the different drugs to the Tyrode's solution.

\subsection{Data analysis: optical APs and calcium transients}

ArcLight and GCaMP5G laser confocal fluorescent recordings were analyzed to characterize the properties of the optical APs and $\mathrm{Ca}^{2+}$ transients respectively using the Clampfit10 program (Molecular Devices). APD 90 was determined from the ArcLight recorded optical APs using a previously validated method [13] as the interval between the timing of 50\% maximal upstroke amplitude (timing of phase 0 ) until the time point of $90 \%$ of repolarization. Other parameters that were measured included the 
spontaneous contraction rate and the decay time of the $\left[\mathrm{Ca}^{2+}\right]$ transient, defined as the time interval from the timing of the calcium transient peak to the point where calcium level is reduced to $20 \%$ of peak amplitude.

\subsection{Isometric force measurements}

ECM-EHTs were shipped to Göttingen, Germany at room temperature (overnight shipment) and analyzed after $72 \mathrm{hrs}$ of recovery at $37^{\circ} \mathrm{C}$. The contractile function of ECM-EHTs was measured as described previously [21]. Briefly, EHTs were transferred into organ baths (Föhr Medical Instruments) containing oxygenated Tyrode's solution at $37^{\circ} \mathrm{C}$. Spontaneous beating rates of EHTs were recorded and EHTs were pre-stretched from $\mathrm{L}_{0}$ (slack length) to Lmax (length of maximal force production) according to the Frank-Starling law. Force of contraction (FOC) was then measured under cumulatively increasing extracellular calcium concentrations $(0.2-4 \mathrm{mM})$. In addition, the responses to the $\beta$ adrenergic agonist isoproterenol $(1 \mu \mathrm{M})$ and the muscarinic agonist carbamylcholin $(10 \mu \mathrm{M})$ were investigated at half-maximal calcium concentration $\left(\mathrm{EC}_{50}\right)$.

\subsection{Statistical analysis}

GraphPad Prism6 (GraphPad Software) was used for statistical analysis. Continuous variables are presented as mean \pm SEM. Categorical variables are expressed as frequencies. Indicated sample sizes (n) represent biological replicates. For the confocal optical imaging studies samples size (n) represents the number of analyzed sites within the EHTs from $\geq 5$ independent experiments. Unpaired student t-test was performed for comparison between two groups of continuous variables. Paired student t-test was performed for comparison between two treatments on the same group of continuous variables. When comparing more than 2 groups, repeated-measurements one-way ANOVA was used, followed by Tukey post-hoc analysis. 


\section{Results}

\subsection{Combining cardiac-specific ECM hydrogel with hiPSCs-CMs to derive the ECM-EHTs}

The pcECM hydrogel was produced through the decellularization of porcine cardiac tissue, followed by solubilization of the isolated ECM using pepsin enzymatic digestion. To control the mechanical properties of the hydrogel, it was supplemented with the biopolymer chitosan ( $0.2 \mathrm{gr} / \mathrm{gr}$ ECM) and then crosslinked using genipin (0.01 gr/gr ECM), which is known to crosslink both chitosan and collagen, as previously described [40]. This formulation was previously found [40] to result in mechanical properties that are in the same range as that of the adult heart [42-45].

The ECM-EHTs were generated by combining cardiomyocytes derived from hiPSCs using a monolayer-based directed differentiation system $[13,47]$ with the aforementioned pcECM hydrogel (Fig.1A). The ECM-EHTs were created from relatively purified populations of differentiating hiPSCCMs, comprised of at least $80 \%$ cTnT+ cells (Fig.S1A). The EHTs were condensed as rings within casting molds and started to contract spontaneously and synchronously after approximately $24-48 \mathrm{hrs}$, indicating establishment of electromechanical connections between the hiPSC-CMs within the ECMEHTs (Movie S1). Following matrix condensation (d3), EHTs were transferred to a passive stretcher device (Fig.1A) where they demonstrated continuous spontaneous contraction for up to 6 months, the longest period tested.

After 30d in culture, immunostaining studies targeting the cardiac sarcomeric marker cTnI (Fig.1B), the ventricular marker MLC-2V (Fig.S1B), and the main ECM component collagen-1 (Fig.1B), revealed the integration and organization of the cells within the remodeled matrix. Cardiomyocytes within the ECM-EHT constructs were arranged anisotropically and developed relatively elongated and oriented cell alignments, a phenomenon not observed in two-dimensional tissue cultures (Fig.1B). Histological analysis revealed the typical cell alignment along the axis of the stretch, a phenomenon that was less obvious in EHTs not subjected to stretch (Fig.S1C). 
TEM analysis revealed the presence of cardiomyocytes in a more mature stage of development in EHTs at 30d as compared to cardiomyocytes in 7d EHTs. This was manifested by a more organized sarcomeric pattern with distinguishable aligned Z-bands in the 30d EHTs, whereas the 7d EHT contained cardiomyocytes with only partially organized myofilament bundles and nascent $\mathrm{Z}$ lines (Fig.1C). SEM analysis revealed the integration of the cells within the ECM and the resulting alteration in ECM structure because of the cellular/ECM interactions (Fig.1D and Fig.S1D).

Gene expression analysis using real-time qPCR (Fig.1E) during the course of EHT maturation (comparing ECM-EHTs at days 7 and 30) revealed statistically significance or a trend for upregulation in the expression of genes associated with the contractile apparatus such as TNNT2 (encoding cTnT), MYL2 (MLC-2v, p<0.01), MYH7 (encoding the $\beta$-MHC isoform, $\mathrm{p}<0.05$ ) and MYH6 $(\alpha-\mathrm{MHC}$ isoform). This was associated with an increase in the ratio of expression of $M Y H 7 / M Y H 6$ as with an increase in the ratio of expression of $M Y L 2 / M Y L 7$ (encoding for MLC-2v and MLC-2a respectively, $\mathrm{p}<0.05$ ), both considered signs of maturation. Similarly, gene expression of $C A S Q 2$, encoding for the major sarcoplasmatic-reticulum $\mathrm{Ca}^{2+}$ buffering protein calsequestrin, was also significantly upregulated $(\mathrm{p}<0.05)$, with a similar trend noted in the expression of $C A C N A 1 C$ (encoding for alpha-1 subunit of a voltage-dependent calcium channel). Importantly, the expression levels of the vast majority of these genes (MYL2, TNNT2, CASQ2, and CACNA1C) was markedly elevated in the ECM-EHTs when compared to 2D cultured cells of similar age (Fig.1E). The maturation process was also associated with the downregulation of gene markers of immature cardiomyocytes such as $N K X 2.5(\mathrm{p}<0.05)$.

\subsection{Electrophysiological and calcium-handling assessment of the ECM-EHTs}

Coupling of the cardiomyocyte's electrical excitation with the resulting mechanical contraction is mediated via transmembrane calcium influx leading to calcium release from intracellular stores, a process known as calcium-induced calcium release (CICR). To study the calcium-handling properties 
of the generated ECM-EHTs, we utilized the emerging technology of genetically-encoded calcium indicators (GECIs) $[13,46]$ and performed laser confocal calcium imaging studies to monitor the cardiomyocytes' intracellular $\left[\mathrm{Ca}^{2+}\right]_{\mathrm{i}}$ transients. Lentiviral transduction was used to express the GCaMP5G transgene (under the transcriptional control of the cTnT promoter) in the differentiating hiPSC-CMs. The GCaMP5G-expressing hiPSC-CMs were then used for the creation of the ECMEHTs. This process resulted in robust expression of the GCaMP5G fluorescent indicator protein in the ECM-EHTs cardiomyocytes, as manifested by their bright green fluorescence (Movie S2 and Fig.2A). Notably, the fluorescence levels of the GCaMP5G-expressing EHTs significantly changed during the cardiac contraction-cycle (Movie S2 and Figs.2A-B) with an increase in fluorescence intensity concomitant with the rise in intracellular $\mathrm{Ca}^{2+}$ levels followed by a reduction in fluorescence levels during relaxation.

High-resolution line-scan evaluation of the GCaMP5G-expressing ECM-EHTs, focusing on the cellular level, revealed typical $\left[\mathrm{Ca}^{2+}\right]_{\mathrm{i}}$ transients (Fig.2B). The optical signals obtained were highly stable, allowing continuous recordings of several optically-derived $\left[\mathrm{Ca}^{2+}\right]_{\mathrm{i}}$ transients from the same cell within the EHT over time and the detailed characterization of each transient. We next evaluated the ability to utilize the GCAMP5G-expressing EHTs for drug testing. Initially, we assessed the capability to trace potential chronotropic changes. The addition of $1 \mu \mathrm{M}$ isoproterenol, a $\beta$-adrenergic agonist, resulted in a positive chronotropic effect. This was manifested by a significant increase in the frequency of the optically-derived $\left[\mathrm{Ca}^{2+}\right]_{i}$ transients, in both the line-scan confocal single-cell resolution experiments (Fig.2C, n=48, $\mathrm{P}<0.001$ ) and in lower resolution imaging studies, deriving the average optical signal from the whole EHT (Fig.S2A). Isoproterenol application also caused significant shortening of the optical signal decay time, indicating more rapid relaxation (Fig.2C right-panel, $\mathrm{n}=37$, $\mathrm{P}<0.001$ and Fig.S2B). In contrast, application of the muscarinic agonist carbamylcholine $(1 \mu \mathrm{M})$ led to a significant negative chronotropic effect, as manifested by a significant decrease in the $\left[\mathrm{Ca}^{2+}\right]_{\mathrm{i}}$ 
transients frequency (Fig.2D and Fig.S2C, n=31, P<0.001), but did not have a significant effect on the signal decay time (Fig.2D and Fig.S2D)

Our next aim was to study the effects of drugs that can alter the morphology of the intracellular $\left[\mathrm{Ca}^{2+}\right]_{\mathrm{i}}$ signal and even lead to the development of arrhythmias. Application of the $\mathrm{I}_{\mathrm{Kr}}$ current blocker E4031 (500nM), known to prolong AP duration (APD) and also potentially to generate early-afterdepolarizations (EADs) and triggered-activity (TA), resulted in the development of significant irregularities in the $\left[\mathrm{Ca}^{2+}\right]_{i}$ transient profile (Fig.2E). We also evaluated the effect of ouabain, a sodium/potassium ATPase inhibitor that can cause intracellular calcium overload, the development of delayed-after-depolarizations (DADs) and TA. Application of $1 \mu \mathrm{M}$ of ouabain led to significant $\left[\mathrm{Ca}^{2+}\right]_{\mathrm{i}}$ transients' irregularity and arrhythmogenic activity. This was manifested by the development of doubleand triple-humped $\left[\mathrm{Ca}^{2+}\right]_{\mathrm{i}}$ transients in the line-scan recordings from the hiPSC-CMs at the single-cell resolution within the ECM-EHTs (Fig.2F), and the presence of spatially abnormal diastolic calcium release and TA events identified at lower-resolution imaging experiments (Fig.2G and Movie S2).

To study the electrophysiological properties of the ECM-EHTs we utilized hiPSC-CMs, which were derived from a transgenic hiPSC line [13] that stably expresses the genetically-encoded voltage indicator (GEVI) ArcLight. As shown in Movie S3 and Fig.3A, the fluorescence levels of the ArcLight-expressing hiPSC-CMs within the EHTs changed during the cardiac contraction-cycle, with an increase in fluorescence levels during the repolarized diastolic resting state followed by a decrease in fluorescence intensity concomitant with membrane depolarization throughout the developed AP. To monitor these fluorescent changes, we utilized the line-scan mode (Fig.3B), which allowed the continuous recordings of several optically-derived APs and detailed characterization of each sampled AP. This permitted, for example, evaluation of APD of the sampled optical APs.

The aforementioned ability to monitor optical APs allowed us to use the ArcLight-expressing ECM-EHTs as an experimental platform for drug testing and specifically to monitor drug-induced 
changes in APD. Identifying potential APD changes may be specifically important for modeling of inherited arrhythmogenic syndromes such as the long and short QT syndromes and in the field of cardiac safety pharmacology. To exemplify the latter, we applied specific ion-channel modulators, focusing on the ability to detect alterations in APD. The chosen agents were designed to either block the rapid component of the delayed rectifier potassium current $\left(\mathrm{I}_{\mathrm{Kr}}\right)$ or to augment the late-sodium current component $\left(\mathrm{I}_{\mathrm{NaL}}\right)$. As can be seen in the dose-response curves, application of escalating doses of the $\mathrm{I}_{\mathrm{Kr}}$ blocker E4031 (100nM-1 $\left.\mu \mathrm{M}\right)$ led to significant prolongation of the measured optical APD 90 values of the hiPSC-CMs within the ECM-EHTs as determined by high-resolution line-scan imaging (Fig.3C, n=35). A similar APD prolongation was also observed following E4031 application in wholeEHT imaging studies (Fig.S3A). Finally, treatment of the ECM-EHTs with ATX-II (30nM), known to augment $\mathrm{I}_{\mathrm{NaL}}$, also led to significant $\mathrm{APD}_{90}$ prolongation (Fig.3D, n=20, $\mathrm{P}<0.001$ ).

\subsection{Contractile function of the ECM-EHTs}

As electrical excitation and calcium handling ultimately result in cell contraction, we next evaluated the contractile properties of the generated engineered tissues. Of note, 30d ECM-EHTs could be shipped from Haifa, Israel to Göttingen, Germany without apparent loss of function. The spontaneous beating rate remained stable at $38 \pm 8$ beats $(n=6)$ per minute after recovery at $37^{\circ} \mathrm{C}$. ECM-EHT displayed the Frank-Starling mechanism and a positive inotropic response to increasing extracellular $\mathrm{Ca}^{2+}$ concentrations (Figs.4A-B). Considering a mean cross sectional area of $1.3 \pm 0.1 \mathrm{~mm}^{2}$ the maximal specific force of contraction (FOC) of ECM-EHT amounted to $0.46 \pm 0.06 \mathrm{mN} / \mathrm{mm}^{2}(\mathrm{n}=6)$. In comparison the to the contractile measurements in 30d ECM-EHTs described above; early-stage (7d) EHTs, although retaining the positive inotropic response to increasing extracellular $\mathrm{Ca}^{2+}$, displayed markedly lower FOC (Fig.S4). These results, indicates the importance of culture time in the process of ECM-EHT maturation. 
We next evaluated the effects of pharmacological agents that could alter contractility. In line with the calcium handling data, application of $1 \mu \mathrm{M}$ isoproterenol increased FOC of the tissue by $42 \%$ and significantly shortened both contraction and relaxation times of the ECM-EHT (Figs.4D-F). The addition of the muscarinic agonist carbamylcholine $(10 \mu \mathrm{M})$ following isoproterenol application induced an antagonistic effect by reducing the isoproterenol-induced increase in FOC by $\sim 10 \%$ (Fig.4D). In contrast the effect of carbamylcholine in reversing isoproterenol-induced shortening of contraction and relaxation times was only minimal and was not statistically significant (Figs.4E-F).

\subsection{Modeling of inherited arrhythmogenic disorders}

To evaluate the ability of the ECM-EHT model to serve as a platform for disease modeling, we established EHTs using patient/disease-specific hiPSC lines derived from patients inflicted with two types of inherited arrhythmogenic syndromes. Initially we created ECM-EHTs using hiPSC-CMs derived from a hiPSC line generated from a young patient with the long QT syndrome type 2 (LQTS2) [9]. Prior to ECM-EHT generation, we used lentiviral transduction to express ArcLight in the LQTS2hiPSC-CMs. We then performed laser confocal line-scans characterizing the AP properties of cardiomyocytes within the diseased LQTS2-ECM-EHTs and compared them to healthy-control ECMEHTs. These optical recordings revealed the typical prolonged APs associated with the LQTS in the LQTS2-ECM-EHT (Figs.5A-B and Fig.S3B), which were significantly longer than the APD values measured in the healthy-control EHTs $(\mathrm{p}<0.001, \mathrm{n}=25)$.

To evaluate the ability to monitor calcium-handling abnormalities, we created ECM-EHTs from hiPSC-CMs derived from a patient, diagnosed with catecholaminergic polymorphic ventricular tachycardia type 2 (CPVT2) [14]. CPVT2 is a familial autosomal recessive arrhythmogenic disorder resulting from homozygous mutations in the $C A S Q 2$ gene encoding the main sarcoplasmic-reticulum (SR) calcium buffer protein calsequestrin [49]. This genetic-abnormality leads to unstable SR calcium 
storage and diastolic calcium leak, which can cause DADs, malignant ventricular arrhythmias, and sudden cardiac death. Following creation of the CPVT2-ECM-EHTs, they were loaded with the calcium-sensitive dye fluo4. We then performed laser-confocal calcium-imaging of fluo4-loaded hiPSC-CMs within the CPVT2-ECM-EHTs and compared the results with similar experiments performed in healthy-control ECM-EHTs. Since arrhythmias in CPVT are often triggered by exercise or emotional stress, the EHTs were treated with isoproterenol $(10 \mu \mathrm{M})$ or evaluated following rapid pacing. As shown in Figs.5C-F, these conditions resulted in the development of significant $\left[\mathrm{Ca}^{2+}\right]_{\mathrm{i}}$ transient abnormalities and arrhythmias characterized by broad double-humped transients in $19.1 \%$ of the CPVT-EHTs. In contrast, only $2.7 \%$ of healthy-control EHTs displayed any calcium transient irregularities (Figs.5C-F, n=50, $\mathrm{P}<0.001$ ). Interestingly, the rate of arrhythmic events were significantly higher in CPVT2-hiPSC-CMs studied as dispersed single-cells when compared to the same cells within the generated EHT environment (Fig.5F).

\subsection{Optical mapping of EHTs for assessment of conduction and reentrant arrhythmias}

Most studies, to date, examined the electrical properties of the hiPSC-CMs at the single-cell level, focusing primarily on AP and ionic-current characteristics. Only a paucity of studies have utilized multicellular hiPSC-based cardiac-tissue models to evaluate conduction and arrhythmogenesis and the majority of these studies focused on two-dimensional monolayer-based models [29, 50-52]. Here we aimed to move the field forward by studying the aforementioned electrophysiological properties also in hiPSC-derived engineered cardiac-muscle tissues. To this end, ECM-EHTs were loaded with the voltage-sensitive dye Di-4-ANBDQBS and optically mapped using a highly-sensitive EM-CCD camera with high spatial and temporal resolutions. The results of these optical mapping studies, performed during both spontaneous activity and point electrical stimulation, verified the development of a functional syncytium within the ECM-EHTs, enabled monitoring of AP wavefront propagation (Movie 
S4), and allowed the construction of detailed activation maps (Figs.6A-C), from which the conduction velocity (CV) of the studied tissues could be measured (Fig.7A). Consequentially, the ECM-EHTs spontaneous activation rate was $0.70 \pm 0.08 \mathrm{~Hz}$ and the measured average $\mathrm{CV}$ in these tissues was found to be $8.0 \pm 0.6 \mathrm{~cm} / \mathrm{s}(\mathrm{n}=13)$.

To monitor the effects of various interventions on the electrophysiological properties of the ECM-EHTs, we first characterized the effects of altering pacing frequency $(0.5$ to $3 \mathrm{~Hz})$ on the tissues conduction properties (Movie S4). Note in the resulting CV restitution plot (Fig.7A), the expected conduction slowing $(\mathrm{P}<0.05, \mathrm{n}=10)$ observed with higher pacing frequencies. Interestingly, at high pacing frequency, we were also able to trigger arrhythmias in $58 \%$ of the ECM-EHTs $(n=12)$. These arrhythmias were all macro-reentrant in nature with a mean rotation frequency of $1.6 \pm 0.2 \mathrm{~Hz}$. Note in Fig.6C, the development of a typical anatomical reentry with the activation wavefront circulating around the ring. In $85 \%$ of the ECM-EHTs developing reentrant arrhythmias, we were able to terminate the arrhythmias and resume normal AP propagation through the application of field stimulation ("electrical shock") (n=7, Movie S5).

We next studied the effect of drugs on the electrophysiological properties of the EHTs. The drugs tested included agents known to slow conduction via two different mechanisms: (1) lidocaine $(100 \mu \mathrm{mol} / \mathrm{L})$, a class Ib anti-arrhythmic agent that blocks the fast voltage-gated $\mathrm{Na}^{+}$channels, and (2) carbenoxolone $(50 \mu \mathrm{mol} / \mathrm{L})$, a gap junction blocker. Studies were performed at a fixed continuous pointstimulation pacing frequency of $1 \mathrm{~Hz}$. Application of lidocaine significantly slowed conduction, as manifested by an increase in the total tissue activation time (Fig.7B). This slowing of conduction was manifested in all ECM-EHTs with an average decrease in the mean CV by $38 \%(\mathrm{P}<0.05, \mathrm{n}=5$, Fig.7B). In a similar manner application of the gap junction uncoupler carbenoxolone also led to significant slowing of conduction with $\sim 50 \%$ reduction in the average $\mathrm{CV}$ values when compared to the baseline values (Fig.7C, n=5, P<0.01). Finally, we also tested the effects of quinidine $(0.1-30 \mu \mathrm{M})$, a $\mathrm{Na}^{+}$ 
channel blocker that also indirectly inhibits potassium currents, and therefore can potentially affect both conduction and repolarization. As can be appreciated from the resulting dose-response curves and activation maps, escalating doses of quinidine significantly prolonged APD 90 values in the EHTs while simultaneously slowing conduction (Fig.7D, $\mathrm{n}=4,{ }^{*} \mathrm{p}<0.05, * * \mathrm{p}<0.01, * * * \mathrm{p}<0.001$ ).

Focusing further on disease modeling of inherited arrhythmogenic disorders, we evaluated the potential arrhythmogenicity of the LQTS2-ECM-EHTs. Interestingly, at baseline the LQTS2-EHTs did not display arrhythmogenic activity, in a similar manner to control ECM-EHT (Fig.7E left-panel and Fig.7F). We then treated the tissue with the Ikr blocking anti-arrhythmic agent dofetilide (25nM). The application of dofetilide led to the development of different types of reentrant arrhythmic activity in the vast majority $(75 \%, \mathrm{n}=8)$ of the LQTS2-EHTs studied (Fig.7E right-panel, Fig.7F, and Supplementary Movie 6). In contrast only $12.5 \%(n=8)$ of control ECM-EHTs tested developed arrhythmic activity following dofetilide application (Fig.7F). 


\section{Discussion}

One of the main objectives of cardiac tissue engineering is to create an in-vitro muscle tissue surrogate of human heart tissue for physiological studies, disease modeling, and drug testing applications [16, 17, 21-29]. The introduction and use of the EHT hydrogel technology to achieve the aforementioned goals was described in several studies, including those involving the use of hPSC-CMs embedded in collagen or fibrin hydrogels. The generated EHTs in these studies displayed cardiac-specific molecular, ultrastructural, and functional properties and were demonstrated to be highly valuable for studying basic muscle physiology, for modeling acquired and inherited cardiac disorders (focusing on different cardiomyopathies), and for diverse drug screening and target validation applications [16, 17, 21-29].

In this study, we aimed to further advance the field by successfully establishing a novel EHT model through the use of a chitosan-enhanced cardiac-specific ECM hydrogel, hiPSCs-CMs derived from patients with inherited arrhythmogenic disorders and healthy-controls, genetically-encoded calcium and voltage indicators, and unique optical mapping technologies. The idea was to utilize tissue engineering components that will be as similar as possible to the native heart. Consequentially, a hydrogel approach was used in which human cardiomyocytes were embedded within a decellularized pcECM hydrogel that was shown previously to preserve many of the heart's ECM original components such as collagen I and III and glycosaminoglycans [40]; thereby providing the cardiomyocytes with the optimal environment [53].

We chose to use pig hearts for this proof-of-concept study as their ECM components are very similar to the human heart and since they were shown not to be immunogenic in our previous study [40]. The use of the cardiac ECM in a form of hydrogel, as an alternative to previously used decellularized heart tissues serving as fixed scaffolds for re-seeding of cells [35-39], has the advantage of allowing to create tissues in any desirable shape, form and dimensions and also the ability to stretch, deform, and stimulate the tissue. Moreover, through the addition of genipin and chitosan in varying 
concentrations, the desired mechanical properties of the generated tissues can be designed and tailored for specific applications. Consequentially, in the current study we utilized the pcECM gel formulation that was supplemented with genipin $(0.01 \mathrm{gr} / \mathrm{gr})$ and with $0.2 \mathrm{gr} / \mathrm{gr}$ chitosan. We chose this specific formulation since in our previous study [40] it displayed mechanical properties that were in the same range of the adult heart [42-45]. Another advantage of the pcECM is the unique decellularization procedure used for its derivation, which avoids the use of strong detergents such as sodium dodecyl sulfate (SDS), thus minimizing the damage to the ECM and preserving its natural composition.

Beyond the use of the decellularized cardiac-specific ECM hydrogel, our study presented several additional novel concepts. These included, the detailed evaluation of conduction and arrhythmogenesis (reentry circuits) in three-dimensional engineered heart tissues and the use of functional fluorescent reporters (GEVIs and GECIs) for long-term repeated assessment of calcium-handling, action potential, and conduction properties of the studied tissues. The development of the aforementioned tools then allowed to establish, for the first time, patient/disease-specific hiPSC-based EHT models of familial arrhythmogenic syndromes such as the LQTS and CPVT and also revealed the value of this model in the field of safety pharmacology. Importantly, the clinical relevance of the established model was highlighted by the finding that the arrhythmogenic potential of the generated CPVT and LQTS tissues was reduced compared to similar single-cell models and by the ability to recapitulate the clinical scenario of pro-arrhythmia in LQTS patients challenged with QT prolonging drugs.

Immunostaining and TEM analysis revealed that the cardiomyocytes within the ECM-EHT are aligned anisotropically, most probably as a consequence of the geometrical forces employed by the passive stretch [54]. The hiPSC-CMs in the current model also developed an elongated shape and more mature sarcomeres; properties that were more advanced when compared to two-dimensional culturing of the same hiPSC-CMs. In a similar manner to other previously described EHT models, assessments of the contractile properties of the ECM-EHTs confirmed the presence of cardiac muscle specific 
behavior, including the well-described length-tension relationship (Frank-Starling law) [55] and appropriate inotropic responses to increases in extracellular calcium as well as to drugs such as isoproterenol and carbamylcholine. Although not the focus of this study, the marked changes observed in 30d vs. 7d ECM-EHTs in the gene expression patterns, morphological and ultrastructural properties, and in the measured FOC all point to the importance of culture time and mechanical conditioning in the maturation process of the ECM-EHTs. Future studies will have to determine whether similar changes can also be observed in the electrophysiological, calcium-handling and metabolic properties of the tissues.

Our study highlights the potential of the ECM-EHT model for drug testing and disease modeling. In the current study we placed a specific emphasis on the electrophysiological aspects associated with such applications. To this end, we combined the ECM-EHT model with genetically encoded calcium and voltage indicators such as GCaMP5G and ArcLight $[13,46]$. These fluorescent protein reporters are comprised of a sensing element such as voltage or calcium sensors that are fused/coupled with a fluorescent reporter element such as circular eGFP. Consequentially, any change in the membrane potential or intracellular $\mathrm{Ca}^{2+}$ levels will lead to a conformational change in the respective sensing element eventually altering the emission fluorescence of the reporter element. The advantage of using GEVIs and GECIs, in contrast to direct electrical recording using intracellular [9] or extracellular electrodes [3] or to the use of voltage and calcium sensitive dyes, stems from their non-invasive nature, minimal cellular toxicity, high signal-to-noise ratio, and long-term expression in the targeted cells. These properties are ideal for applications requiring high throughput screening or continuous and repeated long-term functional phenotyping [13, 29].

Using the aforementioned approach we were able to characterize the electrophysiological and calcium-handling properties of the different ECM-EHTs at the cellular (using laser-confocal microscopy) and tissue (using a specially-designed optical mapping system) resolutions. Initially, we 
focused on assessing the cellular phenotypic properties of hiPSC-CMs within the EHTs using the linescan mode of the confocal microscope and observed typical optical APs and intracellular $\left[\mathrm{Ca}^{2+}\right]_{\mathrm{i}}$ transients. Pharmacological studies demonstrated the ability of such assays to monitor drug-induced changes in these signals in term of contraction rate (isoproterenol, carbamylcholine), changes in the signals morphology (E-4031, ATX2, isoproterenol, and ouabain), and cellular arrhythmogenicity (E403, ouabain).

Similar proof-of-concept experiments revealed the value of such assays also in the field of disease modeling. To this end, we utilized previously established LQTS2 and CPVT2 patient-specific hiPSC-CMs, both displaying a highly arrhythmogenic phenotype at the single-cell level [9, 13, 14]. Our results show that the clinical phenotypes of the LQTS and CPVT; namely AP prolongation and abnormal calcium-handling respectively, can also be identified in the patient/disease-specific ECMEHTs. Interestingly, the arrhythmogenic activity identified in these tissue-constructs was not as frequent, complicated, or severe as previously reported for these cells at the single-cell level. This suggests that the multicellular nature of the generated tissues is associated with an anti-arrhythmic property, probably stemming from electrotonic interactions between the connected cardiomyocytes. This finding is also relevant clinically, as the extremely high incidence of arrhythmias observed in isolated single-cells is probably incompatible with life. Similarly, reentrant arrhythmias were not observed in the LQTS2-ECM-EHTs at baseline but developed in most tissues following treatment with the Ikr blocking anti-arrhythmic agent dofetilide; simulating the clinical scenario of a long QT patient clinically challenged with a QT prolonging agent.

To study complex electrophysiological phenomena such as conduction and reentrant arrhythmic activity at the tissue level, we utilized a high-resolution optical mapping system. These studies revealed the development of a functional cardiac syncytium within the ECM-EHTs, with the electrical activation initiating at the site of a spontaneously generated pacemaker or from the electrode-induced pacing site 
and then propagating to synchronously activate the rest of the tissue. Interestingly, CV values measured in the ECM-EHTs were higher than previously reported in monolayer- or EB- based models [27, 29], indicating a higher degree of electrophysiological maturation and tissue structural organization. Moreover, a typical CV restitution curve was identified in the ECM-EHTs, demonstrating slowing of conduction during faster pacing rates.

Optical mapping of the ECM-EHTs also allowed assessment of the effects of pharmacological interventions on conduction, with agents affecting membrane excitability (lidocaine or quinidine, sodium channel blockers) or cell-to-cell electrical coupling (carbenoxolone) significantly slowing CV in the ECM-EHT model. Interestingly, quinidine, due to its known modulating effects on multiple ionic currents, affected both the tissues' conduction (slower CV at high doses) and repolarization (APD prolongation) properties. Another important application of the new EHT model, is the ability to study reentrant cardiac arrhythmias. By using programmed electrical stimulation, we could induce the development of macro-reentrant arrhythmias in the majority of the EHTs tested, which could be mapped and analyzed. The majority of these arrhythmias could then be converted to normal rhythm by the application of a prolonged electrical field potential, mimicking clinical "electrical cardioversion".

The new ECM-EHT model also possesses certain limitations. The hiPSC-CMs do not display the same level of maturity as cardiomyocytes from the adult heart and this is reflected also in the EHTs' less mature ultrastructural properties, relatively slow $\mathrm{CV}$, and lower mechanical forces generated as compared to the adult heart [56]. For example, the forces recorded in this study from the ECM-EHTs are at least one magnitude lower than the forces generated by the adult human heart muscle [56]. The generated forces, however, are in a similar range to other previously reported tissue engineered cardiac muscle models utilizing hPSC-CMs, with some studies reporting higher values $[45,57]$ and other lower or similar forces $[58,59]$. 
In contrast, the effects of various pharmacological agents identified in the ECM-EHT models, such as isoproterenol, carbamylcholine, E-4031, ATX2, oubain and quinidine are similar to the effects observed in adult heart tissue. Also, the clinical phenotypes of the different cardiac inherited arrhythmogenic disorders (LQTS2 and CPVT2) are recapitulated in the patient-specific ECM-EHT models. Finally the contractile properties of the ECM-EHTs display similar fundamental properties to human heart muscle, such as the length-tension relationship and the observed increase in force as a function of extracellular $\mathrm{Ca}^{2+}$ concentrations.

The relative immaturity of hiPSC-CMs is a general challenge in the field [60]. Consequentially ongoing efforts are focusing on identifying methods to further improve the degree of maturity of hiPSC-CMs at the single-cell and tissue levels including through the use of thyroid and glucocorticoid hormones [61], mechanical and electrical training [29, 45, 59], as well as a variety of other interventions [60].

\section{Conclusions}

By combining patient-specific hiPSC-CMs, a cardiac-specific ECM gel, genetically-encoded fluorescent indicators, and unique optical mapping technologies we were able to establish a clinicallyrelevant engineered cardiac-tissue model. The established ECM-EHT model can undergo detailed molecular and ultrastructural phenotyping. The model can also undergo high-resolution, repeated, longterm functional phenotyping, allowing to characterize the electrophysiological, E-C coupling and contractile properties of the generated tissues and the potential impact of different pharmacological interventions and genetic changes. Consequentially, this approach may bring a unique value to the study of inherited and acquired cardiac disorders and to drug development and testing. 
Disclosures: None

Acknowledgments: This study was partially funded by the European Research Council (ERC-2017COG-773181-iPS-ChOp-AF) and by the Joint Lower Saxony (Niedersachsen) - Israeli research projects fund. M.T. and W.H.Z. are supported by SFB 1002 C04 and S01, SFB 937 A18, IRTG 1816 RP12, and DZHK. 


\section{References}

[1] K. Takahashi, K. Tanabe, M. Ohnuki, M. Narita, T. Ichisaka, K. Tomoda, S. Yamanaka, Induction of pluripotent stem cells from adult human fibroblasts by defined factors, Cell 131 (2007) 861-72.

[2] J. Zhang, G.F. Wilson, A.G. Soerens, C.H. Koonce, J. Yu, S.P. Palecek, J.A. Thomson, T.J. Kamp,

Functional cardiomyocytes derived from human induced pluripotent stem cells, Circ Res 104 (2009) e30-41.

[3] L. Zwi, O. Caspi, G. Arbel, I. Huber, A. Gepstein, I.H. Park, L. Gepstein, Cardiomyocyte Differentiation of Human Induced Pluripotent Stem Cells, Circulation 120 (2009) 1513-23.

[4] Y. Shiba, T. Gomibuchi, T. Seto, Y. Wada, H. Ichimura, Y. Tanaka, T. Ogasawara, K. Okada, N. Shiba, K. Sakamoto, D. Ido, T. Shiina, M. Ohkura, J. Nakai, N. Uno, Y. Kazuki, M. Oshimura, I. Minami, U. Ikeda, Allogeneic transplantation of iPS cell-derived cardiomyocytes regenerates primate hearts, Nature 538 (2016) 388-91.

[5] L. Ye, Y.H. Chang, Q. Xiong, P. Zhang, L. Zhang, P. Somasundaram, M. Lepley, C. Swingen, L. Su, J.S. Wendel, J. Guo, A. Jang, D. Rosenbush, L. Greder, J.R. Dutton, J. Zhang, T.J. Kamp, D.S. Kaufman, Y. Ge, J. Zhang, Cardiac repair in a porcine model of acute myocardial infarction with human induced pluripotent stem cell-derived cardiovascular cells, Cell Stem Cell 15 (2014) 750-61.

[6] L. Zwi-Dantsis, I. Huber, M. Habib, A. Winterstern, A. Gepstein, G. Arbel, L. Gepstein, Derivation and cardiomyocyte differentiation of induced pluripotent stem cells from heart failure patients, Eur Heart J 34 (2013) 1575-86.

[7] A. Moretti, M. Bellin, A. Welling, C.B. Jung, J.T. Lam, L. Bott-Flugel, T. Dorn, A. Goedel, C. Hohnke, F. Hofmann, M. Seyfarth, D. Sinnecker, A. Schomig, K.L. Laugwitz, Patient-specific induced pluripotent stemcell models for long-QT syndrome, N Engl J Med 363 (2010) 1397-409.

[8] N. Sun, M. Yazawa, J. Liu, L. Han, V. Sanchez-Freire, O.J. Abilez, E.G. Navarrete, S. Hu, L. Wang, A. Lee, A. Pavlovic, S. Lin, R. Chen, R.J. Hajjar, M.P. Snyder, R.E. Dolmetsch, M.J. Butte, E.A. Ashley, M.T. Longaker, R.C. Robbins, J.C. Wu, Patient-specific induced pluripotent stem cells as a model for familial dilated cardiomyopathy, Sci Transl Med 4 (2012) 130ra47.

[9] I. Itzhaki, L. Maizels, I. Huber, L. Zwi-Dantsis, O. Caspi, A. Winterstern, O. Feldman, A. Gepstein, G. Arbel, H. Hammerman, M. Boulos, L. Gepstein, Modelling the long QT syndrome with induced pluripotent stem cells, Nature 471 (2011) 225-9.

[10] P. Liang, F. Lan, A.S. Lee, T. Gong, V. Sanchez-Freire, Y. Wang, S. Diecke, K. Sallam, J.W. Knowles, P.J. Wang, P.K. Nguyen, D.M. Bers, R.C. Robbins, J.C. Wu, Drug screening using a library of human induced pluripotent stem cell-derived cardiomyocytes reveals disease-specific patterns of cardiotoxicity, Circulation 127 (2013) 1677-91.

[11] F. Stillitano, J. Hansen, C.W. Kong, I. Karakikes, C. Funck-Brentano, L. Geng, S. Scott, S. Reynier, M. Wu, Y. Valogne, C. Desseaux, J.E. Salem, D. Jeziorowska, N. Zahr, R. Li, R. Iyengar, R.J. Hajjar, J.S. Hulot, 
Modeling susceptibility to drug-induced long QT with a panel of subject-specific induced pluripotent stem cells, eLife 6 (2017)

[12] B.J. van Meer, L.G. Tertoolen, C.L. Mummery, Concise Review: Measuring Physiological Responses of Human Pluripotent Stem Cell Derived Cardiomyocytes to Drugs and Disease, Stem Cells 34 (2016) 2008-15.

[13] R. Shinnawi, I. Huber, L. Maizels, N. Shaheen, A. Gepstein, G. Arbel, A.J. Tijsen, L. Gepstein, Monitoring human-induced pluripotent stem cell-derived cardiomyocytes with genetically encoded calcium and voltage fluorescent reporters, Stem Cell Rep 5 (2015) 582-96.

[14] L. Maizels, I. Huber, G. Arbel, A.J. Tijsen, A. Gepstein, A. Khoury, L. Gepstein, Patient-specific drug screening using a human induced pluripotent stem cell model of catecholaminergic polymorphic ventricular tachycardia type 2, Circ Arrhythm Electrophysiol 10 (2017) e004725-e.

[15] P.W. Burridge, Y.F. Li, E. Matsa, H. Wu, S.G. Ong, A. Sharma, A. Holmstrom, A.C. Chang, M.J. Coronado, A.D. Ebert, J.W. Knowles, M.L. Telli, R.M. Witteles, H.M. Blau, D. Bernstein, R.B. Altman, J.C. $\mathrm{Wu}$, Human induced pluripotent stem cell-derived cardiomyocytes recapitulate the predilection of breast cancer patients to doxorubicin-induced cardiotoxicity, Nat Med 22 (2016) 547-56.

[16] F. Weinberger, I. Mannhardt, T. Eschenhagen, Engineering cardiac muscle tissue: a maturating field of research, Circ Res 120 (2017) 1487-500.

[17] B.M. Ogle, N. Bursac, I. Domian, N.F. Huang, P. Menasche, C.E. Murry, B. Pruitt, M. Radisic, J.C. Wu, S.M. Wu, J. Zhang, W.H. Zimmermann, G. Vunjak-Novakovic, Distilling complexity to advance cardiac tissue engineering, Sci Transl Med 8 (2016) 342ps13.

[18] F. Weinberger, K. Breckwoldt, S. Pecha, A. Kelly, B. Geertz, J. Starbatty, T. Yorgan, K.H. Cheng, K. Lessmann, T. Stolen, M. Scherrer-Crosbie, G. Smith, H. Reichenspurner, A. Hansen, T. Eschenhagen, Cardiac repair in guinea pigs with human engineered heart tissue from induced pluripotent stem cells, Sci Transl Med 8 (2016) 363ra148.

[19] L. Gao, Z.R. Gregorich, W. Zhu, S. Mattapally, Y. Oduk, X. Lou, R. Kannappan, A.V. Borovjagin, G.P. Walcott, A.E. Pollard, V.G. Fast, X. Hu, S.G. Lloyd, Y. Ge, J. Zhang, Large cardiac muscle patches engineered from human induced-pluripotent stem cell-derived cardiac cells improve recovery from myocardial infarction in swine, Circulation 137 (2018) 1712-30.

[20] J. Riegler, M. Tiburcy, A. Ebert, E. Tzatzalos, U. Raaz, O.J. Abilez, Q. Shen, N.G. Kooreman, E. Neofytou, V.C. Chen, M. Wang, T. Meyer, P.S. Tsao, A.J. Connolly, L.A. Couture, J.D. Gold, W.H. Zimmermann, J.C. $\mathrm{Wu}$, Human engineered heart muscles engraft and survive long term in a rodent myocardial infarction model, Circ Res 117 (2015) 720-30.

[21] M. Tiburcy, J.E. Hudson, P. Balfanz, S. Schlick, T. Meyer, M.L. Chang Liao, E. Levent, F. Raad, S. Zeidler, E. Wingender, J. Riegler, M. Wang, J.D. Gold, I. Kehat, E. Wettwer, U. Ravens, P. Dierickx, L.W. van Laake, M.J. Goumans, S. Khadjeh, K. Toischer, G. Hasenfuss, L.A. Couture, A. Unger, W.A. Linke, T. Araki, B. Neel, G. Keller, L. Gepstein, J.C. Wu, W.H. Zimmermann, Defined engineered human myocardium 
with advanced maturation for applications in heart failure modeling and repair, Circulation 135 (2017) 183247.

[22] O. Caspi, A. Lesman, Y. Basevitch, A. Gepstein, G. Arbel, I.H. Habib, L. Gepstein, S. Levenberg, Tissue engineering of vascularized cardiac muscle from human embryonic stem cells, Cir Res 100 (2007) 263-72.

[23] A. Eder, I. Vollert, A. Hansen, T. Eschenhagen, Human engineered heart tissue as a model system for drug testing, Adv Drug Deliv Rev 96 (2016) 214-24.

[24] T.J. Cashman, R. Josowitz, B.V. Johnson, B.D. Gelb, K.D. Costa, Human engineered cardiac tissues created using induced pluripotent stem cells reveal functional characteristics of BRAF-mediated hypertrophic cardiomyopathy, PLoS One 11 (2016) e0146697.

[25] N.L. Tulloch, V. Muskheli, M.V. Razumova, F.S. Korte, M. Regnier, K.D. Hauch, L. Pabon, H. Reinecke, C.E. Murry, Growth of engineered human myocardium with mechanical loading and vascular coculture, Circ Res 109 (2011) 47-59.

[26] I.Y. Shadrin, B.W. Allen, Y. Qian, C.P. Jackman, A.L. Carlson, M.E. Juhas, N. Bursac, Cardiopatch platform enables maturation and scale-up of human pluripotent stem cell-derived engineered heart tissues, Nat Commun 8 (2017) 1825.

[27] Z. Ma, N. Huebsch, S. Koo, M.A. Mandegar, B. Siemons, S. Boggess, B.R. Conklin, C.P. Grigoropoulos, K.E. Healy, Contractile deficits in engineered cardiac microtissues as a result of MYBPC3 deficiency and mechanical overload, Nat Biomed Eng 2 (2018) 955-67.

[28] L.A. MacQueen, S.P. Sheehy, C.O. Chantre, J.F. Zimmerman, F.S. Pasqualini, X. Liu, J.A. Goss, P.H. Campbell, G.M. Gonzalez, S.-J. Park, A.K. Capulli, J.P. Ferrier, T.F. Kosar, L. Mahadevan, W.T. Pu, K.K. Parker, A tissue-engineered scale model of the heart ventricle, Nat Biomed Eng 2 (2018) 930-41.

[29] K. Ronaldson-Bouchard, S.P. Ma, K. Yeager, T. Chen, L. Song, D. Sirabella, K. Morikawa, D. Teles, M. Yazawa, G. Vunjak-Novakovic, Advanced maturation of human cardiac tissue grown from pluripotent stem cells, Nature 556 (2018) 239-43.

[30] D. Zhang, I.Y. Shadrin, J. Lam, H.-Q. Xian, H.R. Snodgrass, N. Bursac, Tissue-engineered cardiac patch for advanced functional maturation of human ESC-derived cardiomyocytes, Biomaterials 34 (2013) 5813-20.

[31] Y.W. Chun, D.A. Balikov, T.K. Feaster, C.H. Williams, C.C. Sheng, J.B. Lee, T.C. Boire, M.D. Neely, L.M. Bellan, K.C. Ess, A.B. Bowman, H.J. Sung, C.C. Hong, Combinatorial polymer matrices enhance in vitro maturation of human induced pluripotent stem cell-derived cardiomyocytes, Biomaterials 67 (2015) 5264.

[32] W.H. Zimmermann, C. Fink, D. Kralisch, U. Remmers, J. Weil, T. Eschenhagen, Three-dimensional engineered heart tissue from neonatal rat cardiac myocytes, Biotechnol Bioeng 68 (2000) 106-14.

[33] T.A. Ahmed, E.V. Dare, M. Hincke, Fibrin: a versatile scaffold for tissue engineering applications, Tissue Eng Part B Rev 14 (2008) 199-215. 
[34] M. Rienks, A.P. Papageorgiou, N.G. Frangogiannis, S. Heymans, Myocardial extracellular matrix: An everchanging and diverse entity, Circ Res 114 (2014) 872-88.

[35] J.P. Guyette, J. Charest, R.W. Mills, B. Jank, P.T. Moser, S.E. Gilpin, J.R. Gershlak, T. Okamoto, G. Gonzalez, D.J. Milan, G.R. Gaudette, H.C. Ott, Bioengineering human myocardium on native extracellular matrix, Circ Res 118 (2016) 56-72.

[36] H.C. Ott, T.S. Matthiesen, S.K. Goh, L.D. Black, S.M. Kren, T.I. Netoff, D.A. Taylor, Perfusiondecellularized matrix: using nature's platform to engineer a bioartificial heart, Nat Med 14 (2008) 213-21.

[37] T.Y. Lu, B. Lin, J. Kim, M. Sullivan, K. Tobita, G. Salama, L. Yang, Repopulation of decellularized mouse heart with human induced pluripotent stem cell-derived cardiovascular progenitor cells, Nature communications 4 (2013) 2307.

[38] Q. Wang, H. Yang, A. Bai, W. Jiang, X. Li, X. Wang, Y. Mao, C. Lu, R. Qian, F. Guo, T. Ding, H. Chen, S. Chen, J. Zhang, C. Liu, N. Sun, Functional engineered human cardiac patches prepared from nature's platform improve heart function after acute myocardial infarction, Biomaterials 105 (2016) 52-65.

[39] Y. Eitan, U. Sarig, N. Dahan, M. Machluf, Acellular cardiac extracellular matrix as a scaffold for tissue engineering: in vitro cell support, remodeling, and biocompatibility, Tissue Eng Part C, Methods 16 (2010) 671-83.

[40] Y. Efraim, H. Sarig, N. Cohen Anavy, U. Sarig, E. de Berardinis, S.Y. Chaw, M. Krishnamoorthi, J. Kalifa, H. Bogireddi, T.V. Duc, T. Kofidis, L. Baruch, F.Y.C. Boey, S.S. Venkatraman, M. Machluf, Biohybrid cardiac ECM-based hydrogels improve long term cardiac function post myocardial infarction, Acta Biomater 50 (2017) 220-33.

[41] V. Russo, E. Omidi, A. Samani, A. Hamilton, L.E. Flynn, Porous, Ventricular extracellular matrix-derived foams as a platform for cardiac cell culture, Biores Open Access 4 (2015) 374-88.

[42] M.F. Berry, A.J. Engler, Y.J. Woo, T.J. Pirolli, L.T. Bish, V. Jayasankar, K.J. Morine, T.J. Gardner, D.E. Discher, H.L. Sweeney, Mesenchymal stem cell injection after myocardial infarction improves myocardial compliance, Am J Physiol Heart Circ Physiol 290 (2006) H2196-203.

[43] B. Bhana, R.K. Iyer, W.L. Chen, R. Zhao, K.L. Sider, M. Likhitpanichkul, C.A. Simmons, M. Radisic, Influence of substrate stiffness on the phenotype of heart cells, Biotechnol Bioeng 105 (2010) 1148-60.

[44] A.J. Engler, C. Carag-Krieger, C.P. Johnson, M. Raab, H.Y. Tang, D.W. Speicher, J.W. Sanger, J.M. Sanger, D.E. Discher, Embryonic cardiomyocytes beat best on a matrix with heart-like elasticity: scar-like rigidity inhibits beating, J Cell Sci 121 (2008) 3794-802.

[45] Y. Yahalom-Ronen, D. Rajchman, R. Sarig, B. Geiger, E. Tzahor, Reduced matrix rigidity promotes neonatal cardiomyocyte dedifferentiation, proliferation and clonal expansion, eLife 4 (2015)

[46] R.C. Addis, J.L. Ifkovits, F. Pinto, L.D. Kellam, P. Esteso, S. Rentschler, N. Christoforou, J.A. Epstein, J.D. Gearhart, Optimization of direct fibroblast reprogramming to cardiomyocytes using calcium activity as a functional measure of success, J Mol Cell Cardiol 60 (2013) 97-106. 
[47] P.W. Burridge, E. Matsa, P. Shukla, Z.C. Lin, J.M. Churko, A.D. Ebert, F. Lan, S. Diecke, B. Huber, N.M. Mordwinkin, J.R. Plews, O.J. Abilez, B. Cui, J.D. Gold, J.C. Wu, Chemically defined generation of human cardiomyocytes, Nat Met 11 (2014) 855-60.

[48] M. Tiburcy, T. Meyer, P.L. Soong, W.H. Zimmermann, Collagen-based engineered heart muscle, Methods Mol Biol 1181 (2014) 167-76.

[49] H. Lahat, E. Pras, T. Olender, N. Avidan, E. Ben-Asher, O. Man, E. Levy-Nissenbaum, A. Khoury, A. Lorber, B. Goldman, D. Lancet, M. Eldar, A missense mutation in a highly conserved region of CASQ2 is associated with autosomal recessive catecholamine-induced polymorphic ventricular tachycardia in Bedouin families from Israel, Am J Hum Genet 69 (2001) 1378-84.

[50] T.J. Herron, Calcium and voltage mapping in hiPSC-CM monolayers, Cell Calc 59 (2016) 84-90.

[51] Z. Laksman, M. Wauchop, E. Lin, S. Protze, J. Lee, W. Yang, F. Izaddoustdar, S. Shafaattalab, L. Gepstein, G.F. Tibbits, G. Keller, P.H. Backx, Modeling atrial fibrillation using human embryonic stem cell-derived atrial tissue, Sci Rep 7 (2017) 5268.

[52] S. Kadota, I. Minami, N. Morone, J.E. Heuser, K. Agladze, N. Nakatsuji, Development of a reentrant arrhythmia model in human pluripotent stem cell-derived cardiac cell sheets, Eur Heart J 34 (2013) 1147-56.

[53] G.M. Fomovsky, S. Thomopoulos, J.W. Holmes, Contribution of extracellular matrix to the mechanical properties of the heart, J Mol Cell Cardiol 48 (2010) 490-6.

[54] M. Gonen-Wadmany, L. Gepstein, D. Seliktar, Controlling the cellular organization of tissue-engineered cardiac constructs, Ann New York Acad Sci 1015 (2004) 299-311.

[55] V. Sequeira, J. van der Velden, Historical perspective on heart function: the Frank-Starling Law, Biophys Rev 7 (2015) 421-47.

[56] J. van der Velden, L.J. Klein, M. van der Bijl, M.A. Huybregts, W. Stooker, J. Witkop, L. Eijsman, C.A. Visser, F.C. Visser, G.J. Stienen, Isometric tension development and its calcium sensitivity in skinned myocyte-sized preparations from different regions of the human heart, Cardiovasc Res 42 (1999) 706-19.

[57] A.F.G. Godier-Furnémont, M. Tiburcy, E. Wagner, M. Dewenter, S. Lämmle, A. El-Armouche, S.E. Lehnart, G. Vunjak-Novakovic, W.-H. Zimmermann, Physiologic force-frequency response in engineered heart muscle by electromechanical stimulation, Biomaterials 60 (2015) 82-91.

[58] I. Mannhardt, K. Breckwoldt, D. Letuffe-Breniere, S. Schaaf, H. Schulz, C. Neuber, A. Benzin, T. Werner, A. Eder, T. Schulze, B. Klampe, T. Christ, M.N. Hirt, N. Huebner, A. Moretti, T. Eschenhagen, A. Hansen, Human engineered heart tissue: analysis of contractile force, Stem Cell Rep 7 (2016) 29-42.

[59] J.L. Ruan, N.L. Tulloch, M.V. Razumova, M. Saiget, V. Muskheli, L. Pabon, H. Reinecke, M. Regnier, C.E. Murry, Mechanical stress conditioning and electrical stimulation promote contractility and force maturation of induced pluripotent stem cell-derived human cardiac tissue, Circulation 134 (2016) 1557-67.

[60] X. Yang, L. Pabon, C.E. Murry, Engineering adolescence: Maturation of human pluripotent stem cellderived cardiomyocytes, Circ Res 114 (2014) 511-23. 
[61] S.S. Parikh, D.J. Blackwell, N. Gomez-Hurtado, M. Frisk, L. Wang, K. Kim, C.P. Dahl, A. Fiane, T. Tonnessen, D.O. Kryshtal, W.E. Louch, B.C. Knollmann, Thyroid and glucocorticoid hormones promote functional T-tubule development in human-induced pluripotent stem cell-derived cardiomyocytes, Circ Res 121 (2017) 1323-30. 


\section{Figure Legends}

Figure 1. EHT-ECM generation and characterization.

[A] Schematic representation of the process of ECM-EHT generation by combining hiPSC-CMs with the cardiac-specific ECM gel. Scale: $20 \mu \mathrm{m}$ (left-panel), 0.5mm (right-panel). [B] Immunostaining of the ECM-EHTs for cTnI alone (middle-panel) or together with collagen I (left-panel) in comparison to control hiPSC-CMs grown as two-dimensional cultures (right-panel). Nuclei were counterstained with DAPI. Note that cardiomyocytes in the ECM-EHT are characterized by a more organized anisotropic structure and elongated shape. Scale: $10 \mu \mathrm{m}$. [C] Ultrastructural characterization of the EHT-ECM by TEM showing development of a more mature sarcomeric structures in hiPSCs-CMs within a 30d-EHT as compared to a 7d-EHT. [D] SEM images of an acellular ECM-only scaffold (top) as compared to a

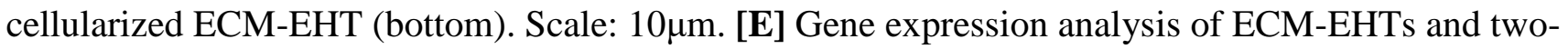
dimension cultured hiPSC-CMs at different maturation stages ( 7 or 30 days, $n=6-7$ ). Note the statistically upregulation over time of genes associated with contractile function (TNNT2, MYH7, MYL2, and MYL2/MYL7 ratio) and excitation-contraction coupling (CASQ2) coupled with the downregulation of early-stage cardiac genes (NKX2.5). ${ }^{*} \mathrm{p}<0.05,{ }^{* *} \mathrm{p}<0.01, * * * \mathrm{p}<0.001$.

\section{Figure 2. Evaluation of calcium-handing in the ECM-EHTs and drug testing}

[A] Changes in the fluorescence levels of GCaMP5G-expressing ECM-EHT during the cardiac contraction-cycle showing the increase in fluorescence during systole (right-panel). Scale: $0.5 \mathrm{~mm}$. [B] High-resolution line-scan imaging showing typical optical intracellular $\left[\mathrm{Ca}^{2+}\right]_{\mathrm{i}}$ transients in sampled hiPSC-CMs within the ECM-EHT. [C-E] Representative line-scan images showing changes in intracellular $\left[\mathrm{Ca}^{2+}\right]_{\mathrm{i}}$ transients at baseline and following applications of $1 \mu \mathrm{M}$ isoproterenol $(\mathrm{C}), 1 \mu \mathrm{M}$ carbamylcholine (D), and 500nM E4031 (E). Also summarized are the changes induced by isoproterenol $(\mathrm{n}=48 ; \mathrm{C})$ and carbamylcholine $(\mathrm{n}=31 ; \mathrm{D})$ in the contraction rates (middle-panels) and in 
the signal decay time (right-panels). $* \mathrm{P}<0.01$. [F-G] Effects of application of $1 \mu \mathrm{M}$ oubain on calciumhandling and arrhythmogenicity in the ECM-EHT at both the cellular level within the EHT (using linescan confocal imaging, F) and at the tissue level (using time-lapse images of the entire GCaMP5expressing EHT). Note the significant drug-induced abnormalities in the $\left[\mathrm{Ca}^{2+}\right]_{\mathrm{i}}$ transients and the significant arrhythmogenic activity (arrows). Scale: $0.5 \mathrm{~mm}$.

\section{Figure 3. Evaluation of AP properties and drug testing using ArcLight imaging}

[A] Changes in the fluorescence levels of ArcLight-expressing ECM-EHT during the cardiomyocyte contraction-cycle. Note the reduction of fluorescence intensity concomitant with membrane depolarization (right-panel). Scale: 0.5mm. [B] Line-scan imaging showing typical ArcLight-based optical AP transients. [C-D] Representative line-scan images of optical APs (left-panels) and summary of $\mathrm{APD}_{90}$ measurements from hiPSC-CMs within the EHTs (right-panels) at baseline and following application of the $\mathrm{I}_{\mathrm{Kr}}$ current blocker E4031 (100nM-1 $\left.\mu \mathrm{M}, \mathrm{n}=35, \mathrm{C}\right)$ or the late-sodium current activator ATX-II (30ng, $\mathrm{n}=20, \mathrm{D}) .{ }^{*} \mathrm{p}<0.05, * * \mathrm{p}<0.01, * * * \mathrm{p}<0.001$

\section{Figure 4. Contractile properties of 30d ECM-EHTs}

[A] Frank-Starling mechanism in ECM-EHT. Representative original trace of increase in developed force of contraction (FOC, gray area) in response to increases in tissue length from L0 (slack length) to Lmax (length of maximal FOC). [B] FOC (normalized to cross sectional area, CSA) of ECM-EHT at

L0 and Lmax, ${ }^{*} \mathrm{p}<0.05$ by Student's t-test. [C] Effects of increasing extracellular $\mathrm{Ca}^{2+}$ concentration on FOC of ECM-EHTs (normalized per cross sectional area, CSA). [D] Changes in FOC (percent change from pre-treatment) of ECM-EHTs after application of $1 \mu \mathrm{M}$ isoproterenol (Iso) followed by addition of $10 \mu \mathrm{M}$ carbamylcholin (Carb). [E-F] Effects of application of $1 \mu \mathrm{M}$ Iso followed by addition of $10 \mu \mathrm{M}$ 
Carb on the ECM-EHTs contraction (T1-90: Time to 90\% contraction (E) and relaxation (T2-50: Time to $50 \%$ relaxation $(\mathrm{F})$ times $(\mathrm{n}=6) .{ }^{*} \mathrm{p}<0.05, * * \mathrm{p}<0.01$.

\section{Figure 5. Functional characterization of LQTS and CPVT2 ECM-EHTs}

[A] Representative line-scan images showing prolongations of the optical APs of hiPSC-CMs within the LQTS2-ECM-EHTs as compared to recordings from healthy-control ECM-EHTs. [B] Changes in $\mathrm{APD}_{90}$ in LQTS2-EHTs compare to healthy-control ECM-EHTs (n=25). *p<0.001. [C-D] Laserconfocal $\left[\mathrm{Ca}^{2+}\right]_{\mathrm{i}}$ imaging of hiPSC-CMs within the healthy-control (C) and CPVT2 (D) ECM-EHTs at baseline, after addition of $10 \mu \mathrm{M}$ isoproterenol, and following rapid pacing $(2 \mathrm{~Hz})$. Note the resulting irregular activity in the cardiomyocytes within the CPVT-ECM-EHTs (arrows) but not in control tissues. [E] Laser-confocal $\left[\mathrm{Ca}^{2+}\right]_{\mathrm{i}}$ imaging of 2D-cultured single-cell dispersed CPVT-hiPSC-CMs at baseline and after addition of $10 \mu \mathrm{M}$ isoproterenol. [F] Quantification of the percentage of cells displaying abnormal $\left[\mathrm{Ca}^{2+}\right]_{\mathrm{i}}$ transients in isolated CPVT-hiPSC-CMs, in hiPSC-CMs within the CPVTEHTs, and in hiPSC-CMs within healthy-control EHTs. ${ }^{*} \mathrm{p}<0.001$.

\section{Figure 6. Optical mapping of the ECM-EHTs}

Sequential images (left-panels) taken from the results of the optical mapping dynamic displays of the ECM-EHTs during spontaneous rhythm (A), during electrical point stimulation (B) and during an induced circulating reentrant arrhythmia (C). The resulting activation maps for each condition are shown in the right-panels. Scale: $0.5 \mathrm{~mm}$.

\section{Figure 7. Evaluating conduction in the ECM-EHTs}

[A] CV restitution curve depicting the typical slowing of conduction in response to increasing stimulation frequency $(n=10)$. [B-C] Examples of activation maps (left and middle panels) of the ECMEHTs and summary of the resulting changes in mean CV values (right-panels) at baseline and following administration of $100 \mu \mathrm{M}$ lidocaine $(\mathrm{n}=5, \mathrm{~B})$ or $50 \mu \mathrm{M}$ of carbenoxolone $(\mathrm{n}=5, \mathrm{C})$. 
[D] Representative activation maps showing the effect of Quinidne $(10 \mu \mathrm{M})$ on the ECM-EHTs conduction (upper-panels). Dose-response curves (lower-panels) showing the effects of escalating doses of quinidine $(0.1,0.3,1,3,10$, and $30 \mu \mathrm{M}, \mathrm{n}=4)$ on APD (left) and CV (right). [E] Examples of activation maps demonstrating the effect of administration of 25nM dofetilide on the LQTS2-ECMEHTs on the development of reentrant arrhythmias. [F[ Quantification of the percentage of healthycontrol and LQTS2 ECM-EHT that developed arrhythmias at baseline and after addition of dofetilide. $* \mathrm{p}<0.05, * * \mathrm{p}<0.01, * * * \mathrm{p}<0.001$ 

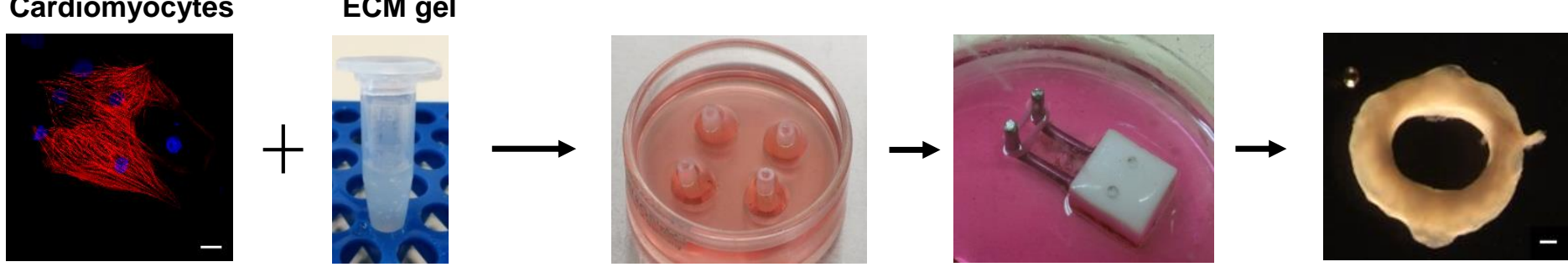

B

cTnl/collagen

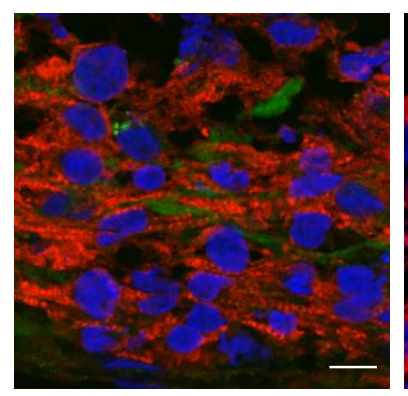

D ECM only

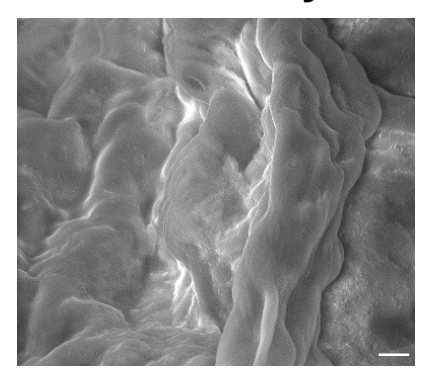

ECM and cells

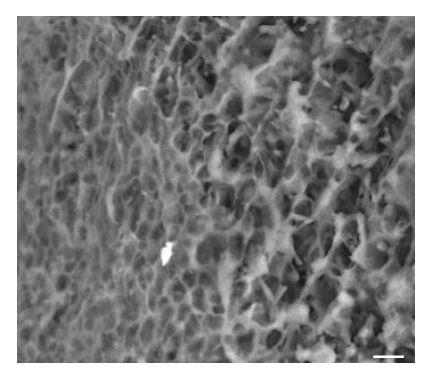

cTnl

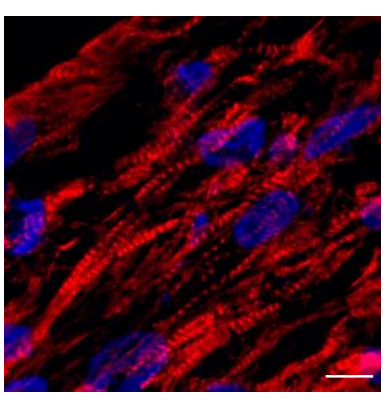

E MYL2

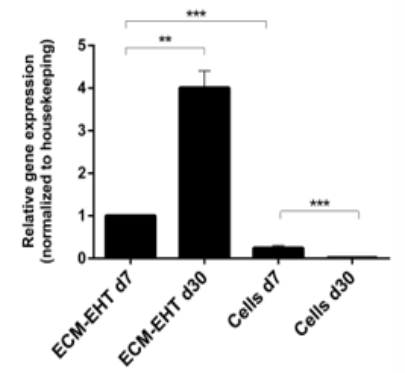

MYH7

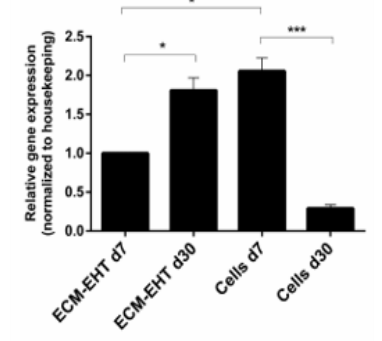

Cells in culture

cTnl

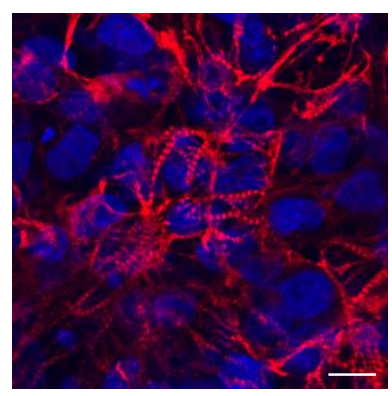

MYL7

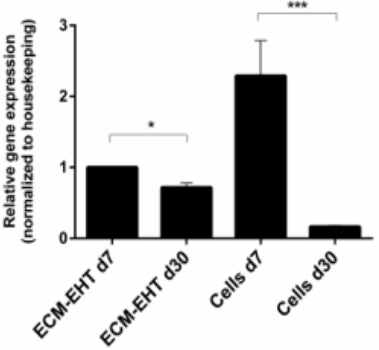

MYH6

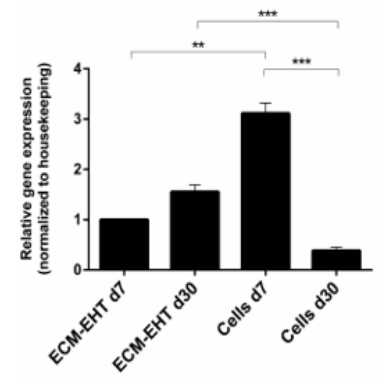

C
EHT 7 day

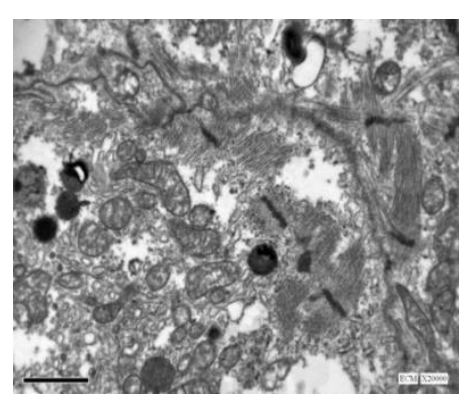

MYL2/MYL7
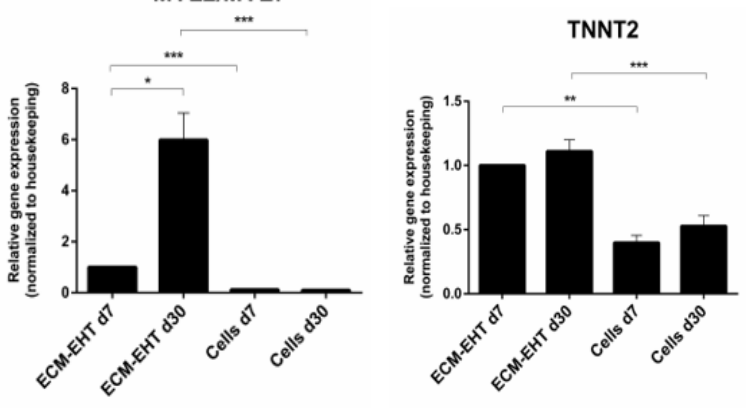

EHT 30 day

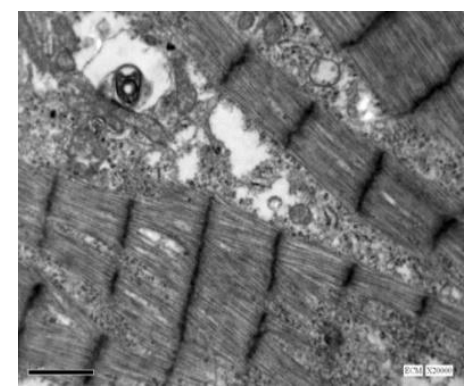

Ping

Nkx2.5

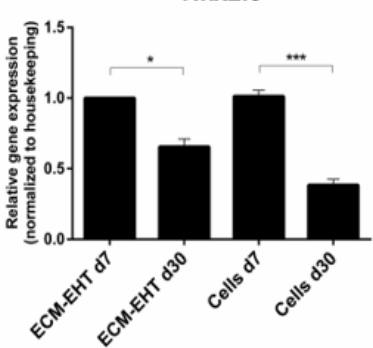

MYH7/ MYH6

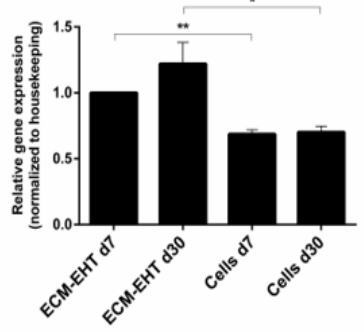

CACNA1C

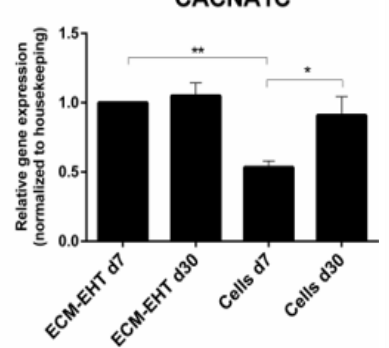

CASQ2

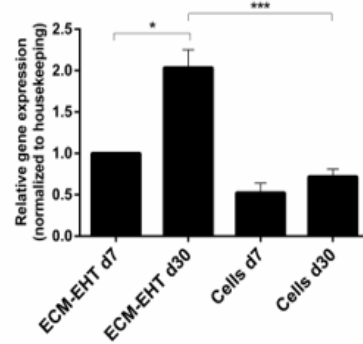


Figure 2

A
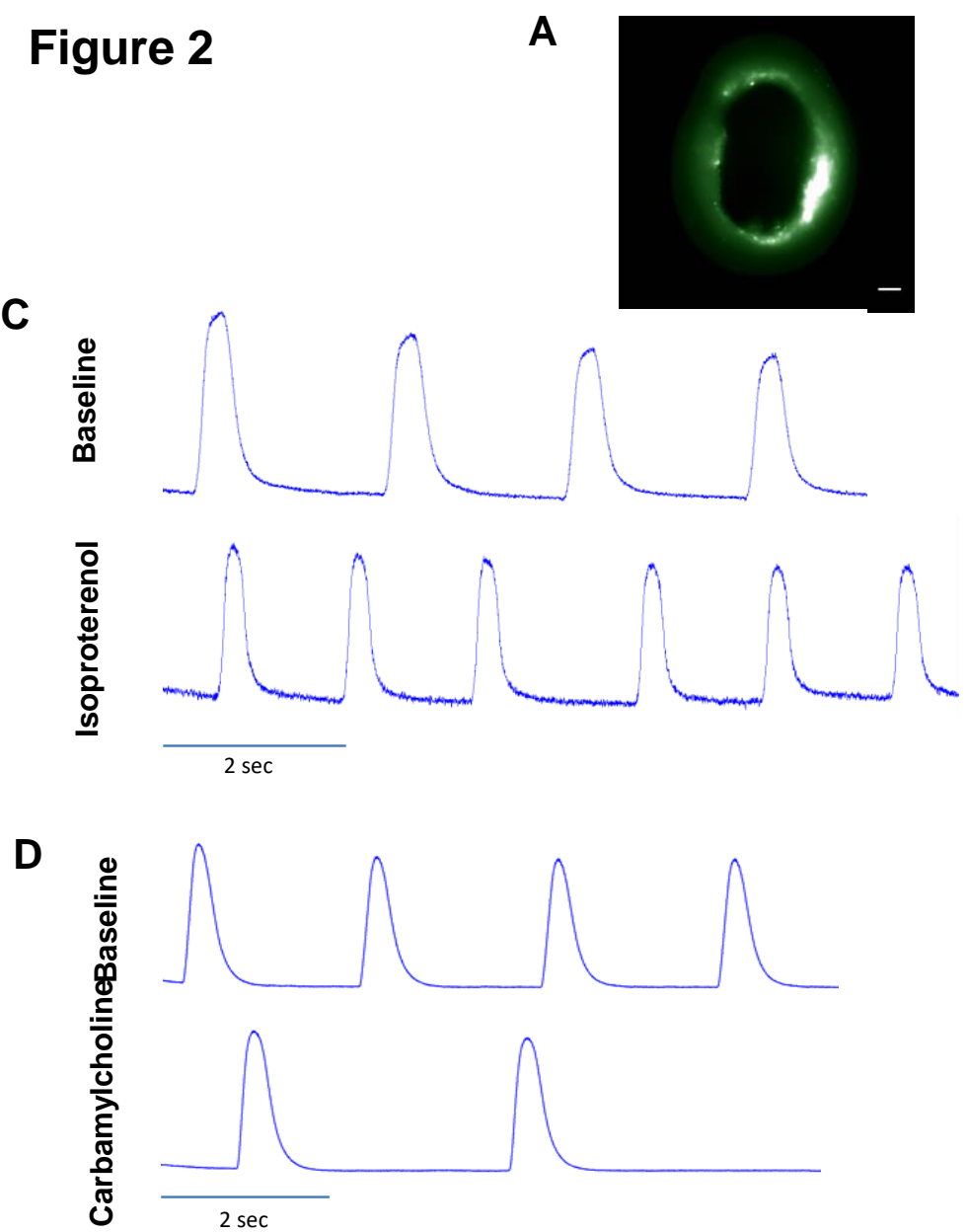

E
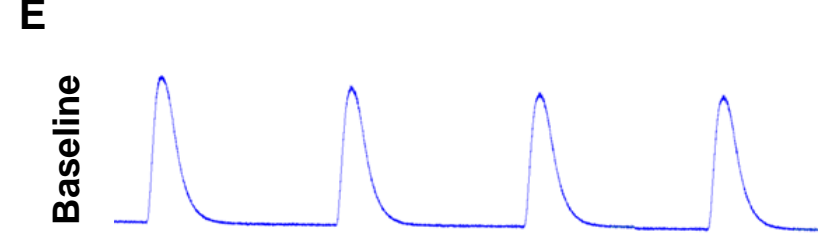

ஜ্ণ
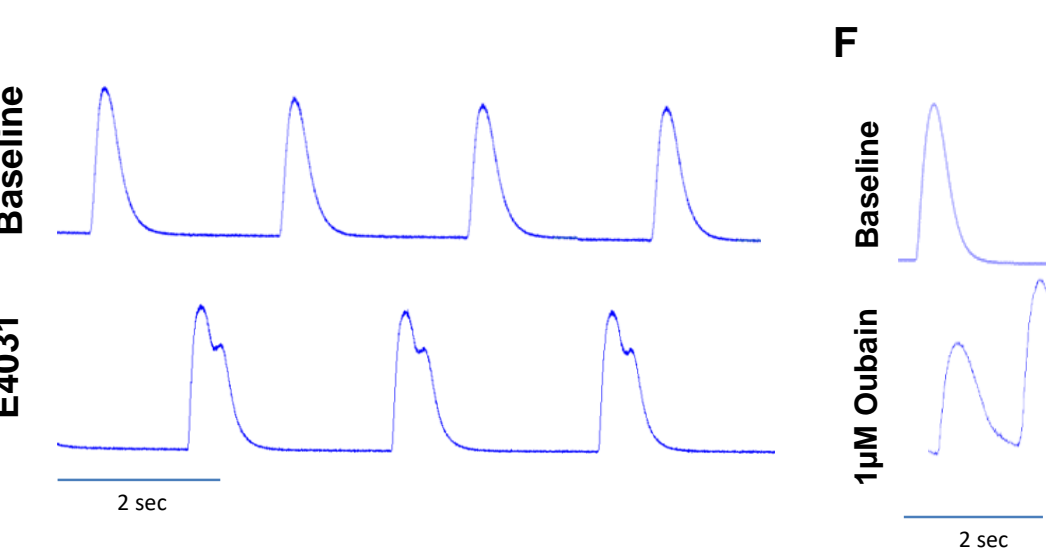
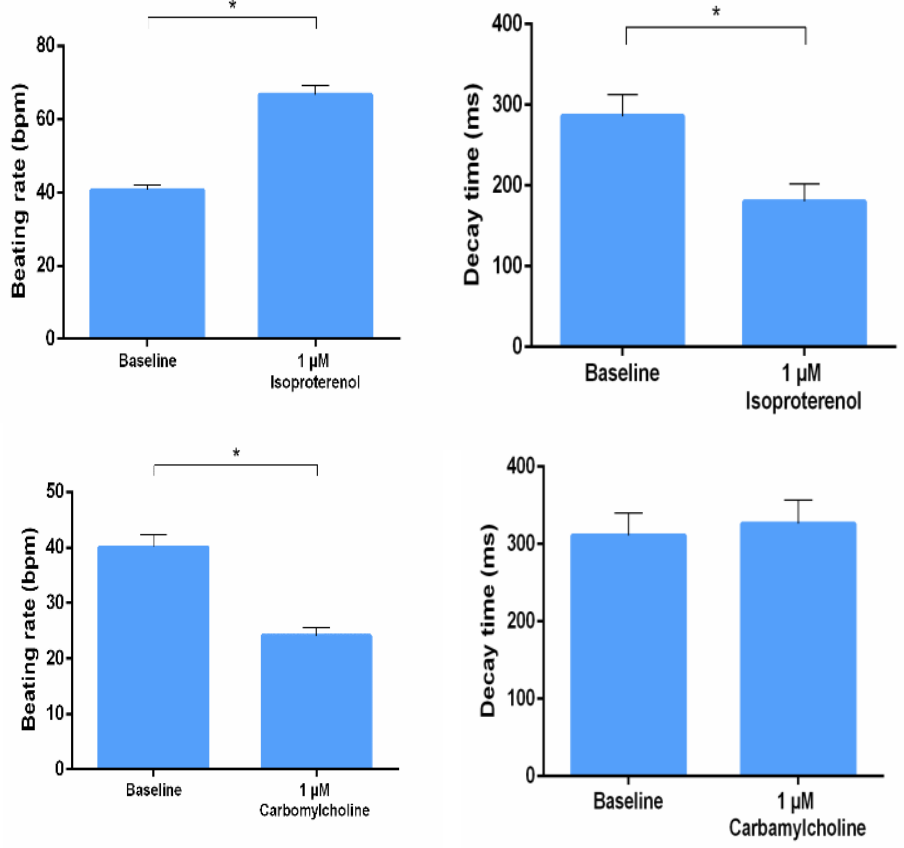

G
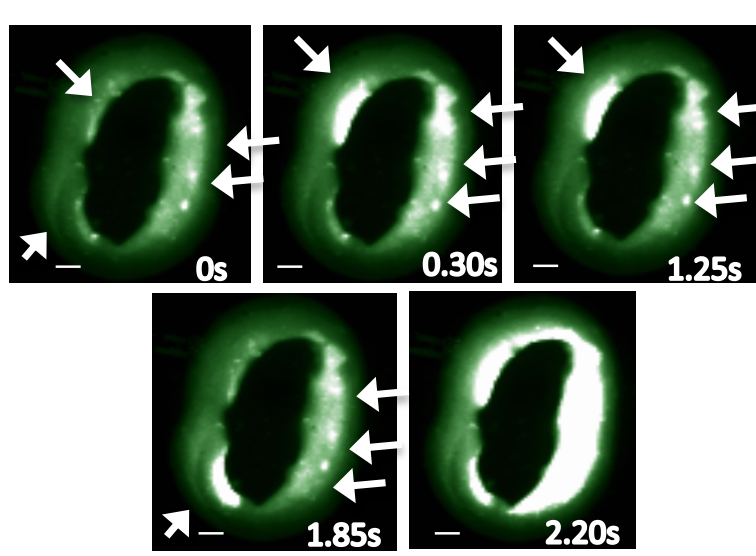
Figure 3

A
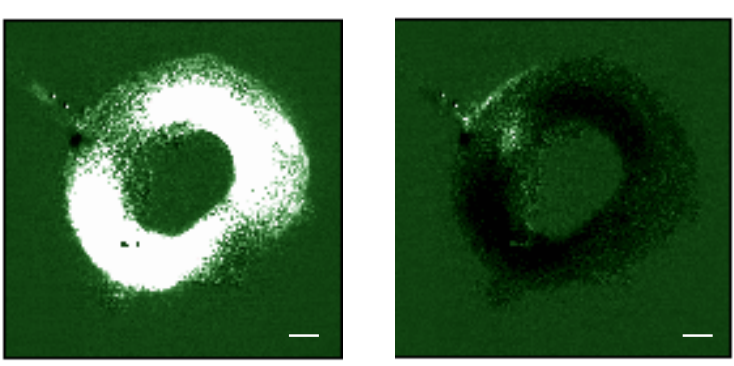

B

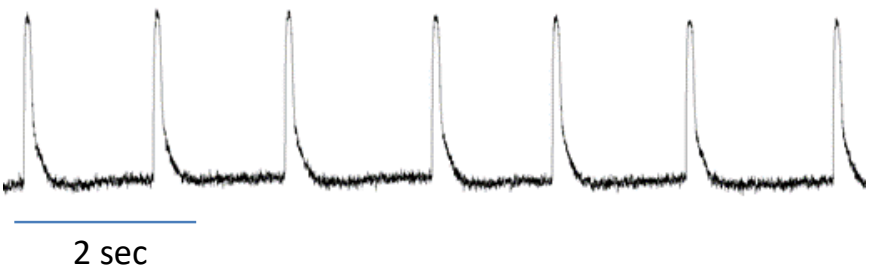

C
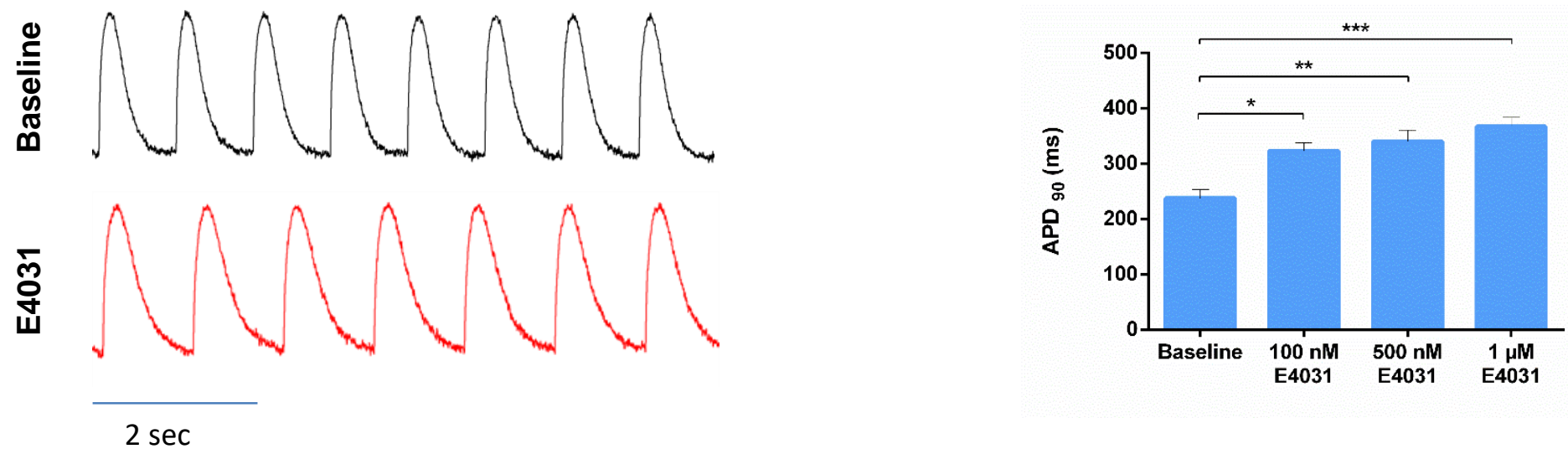

D
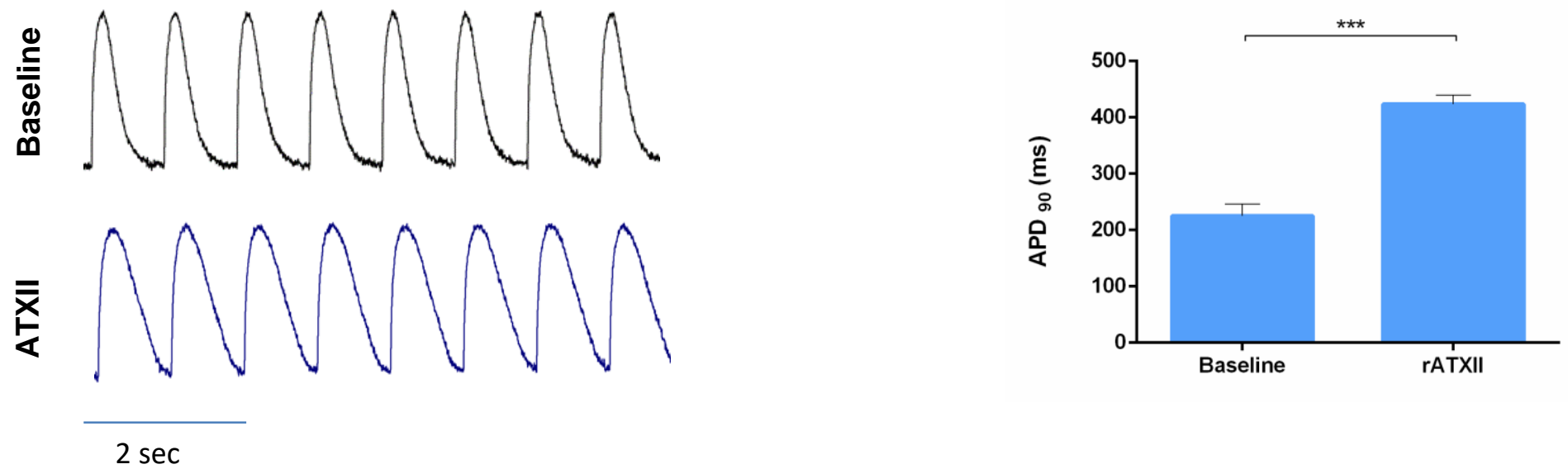


\section{Figure 4}

A

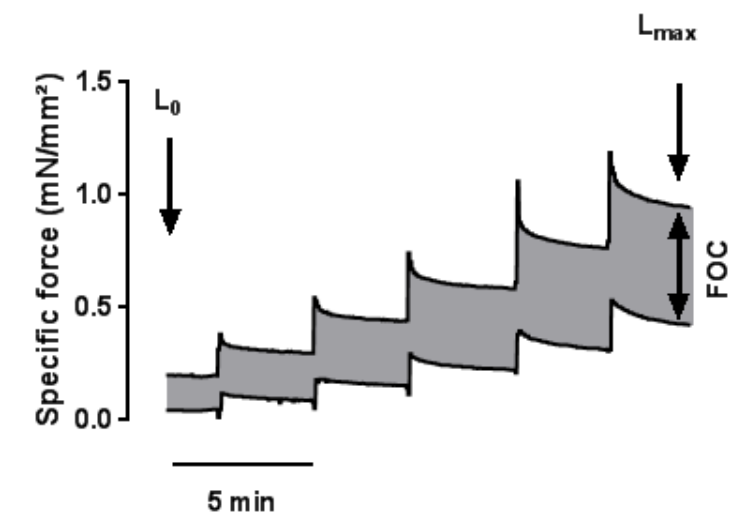

D

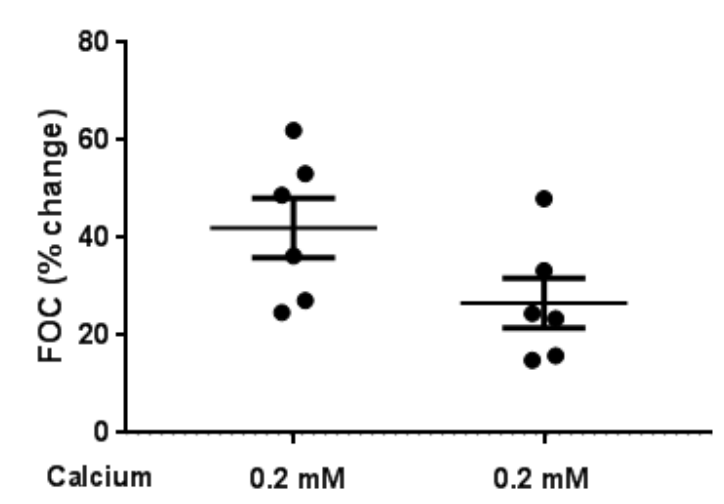

B

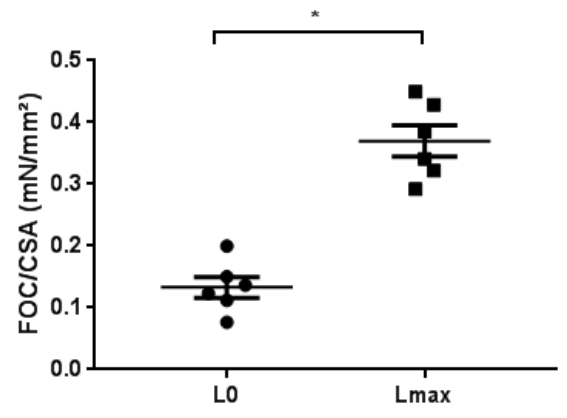

E

Single EHM contraction time

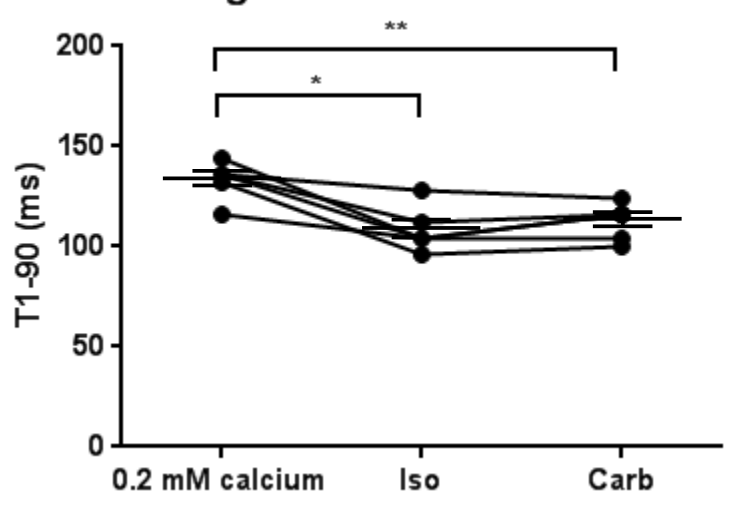

C

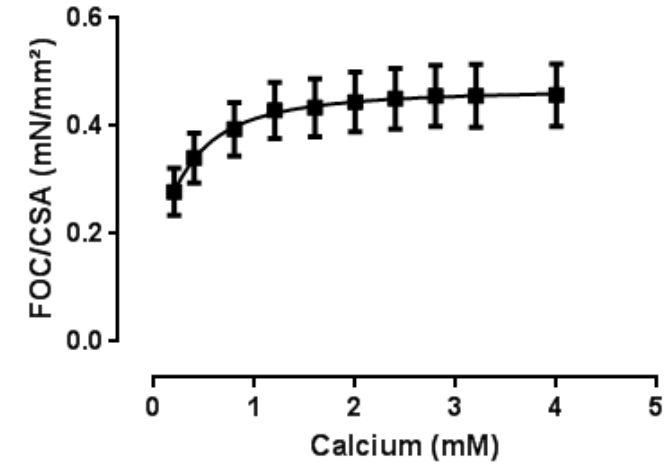

F

Single EHM relaxation times

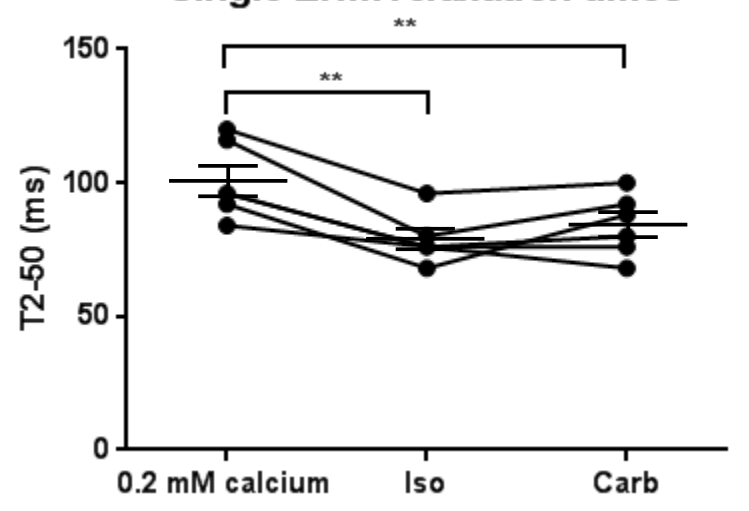




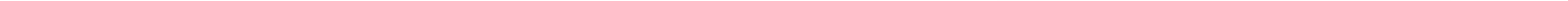




\section{Figure 6}
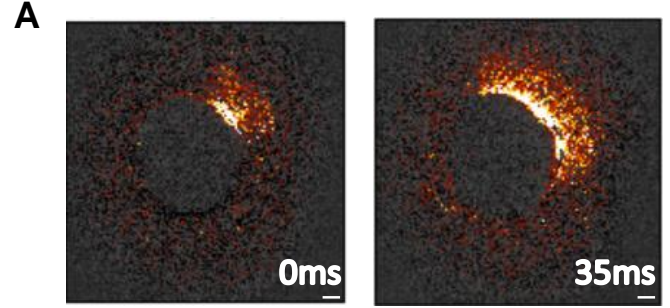

B
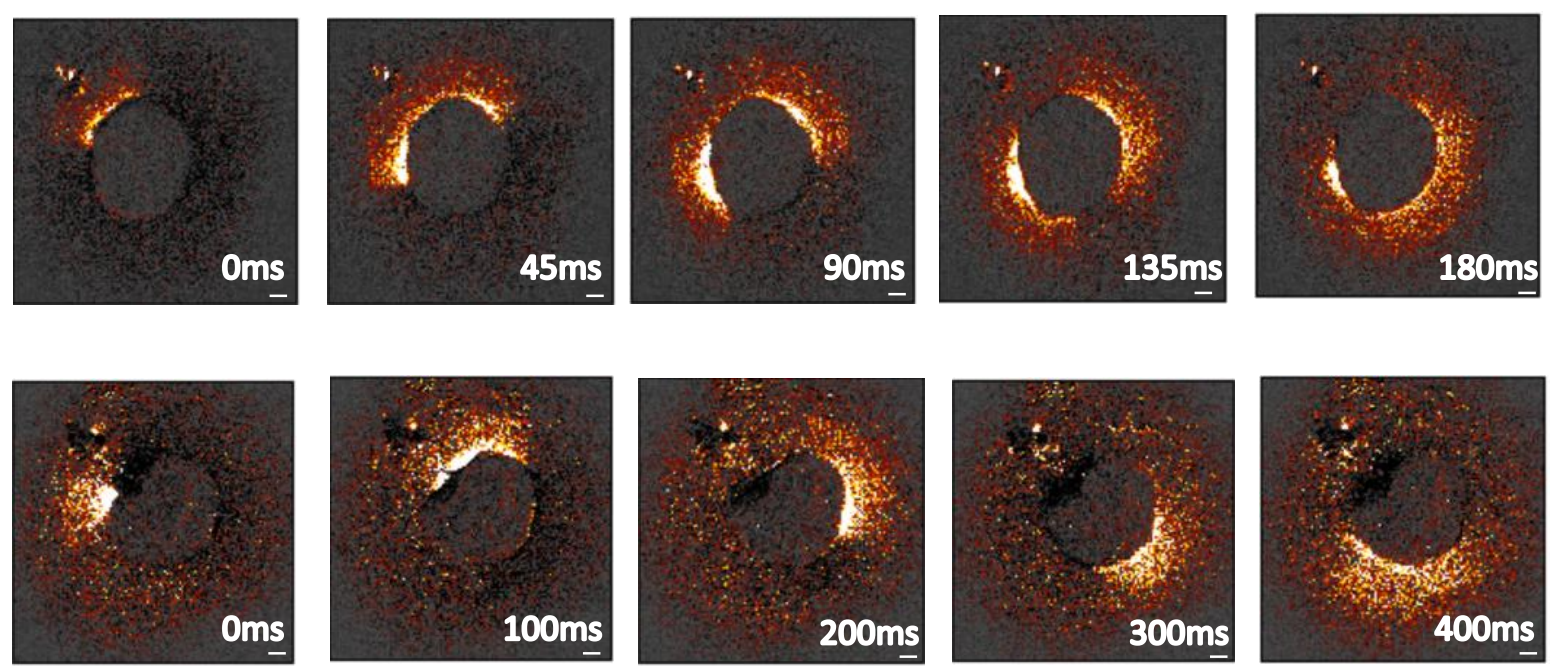
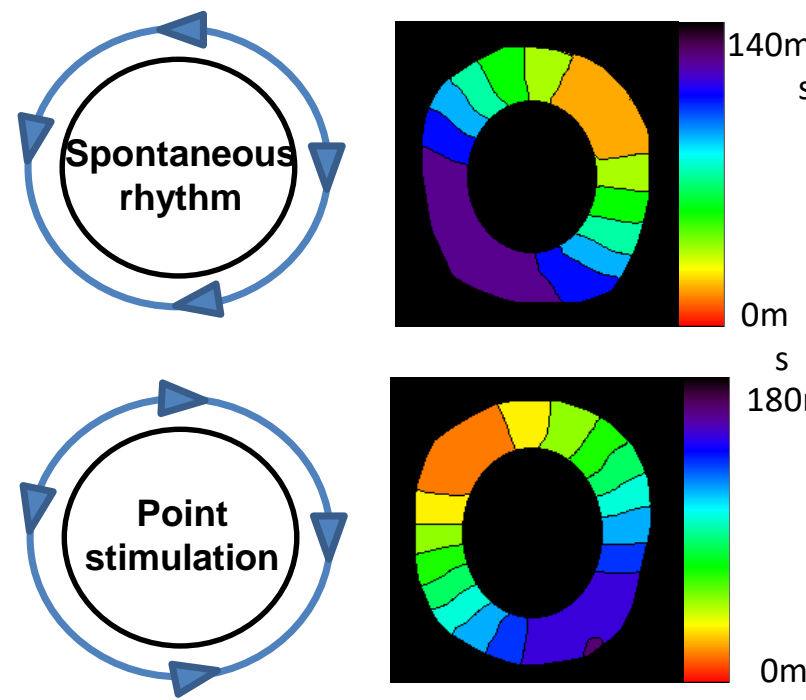

$\mathrm{s}$

$180 \mathrm{~m}$

$\mathrm{s}$
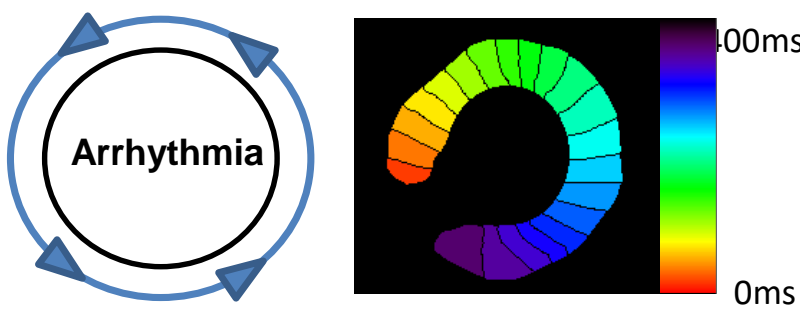
Figure 7

A

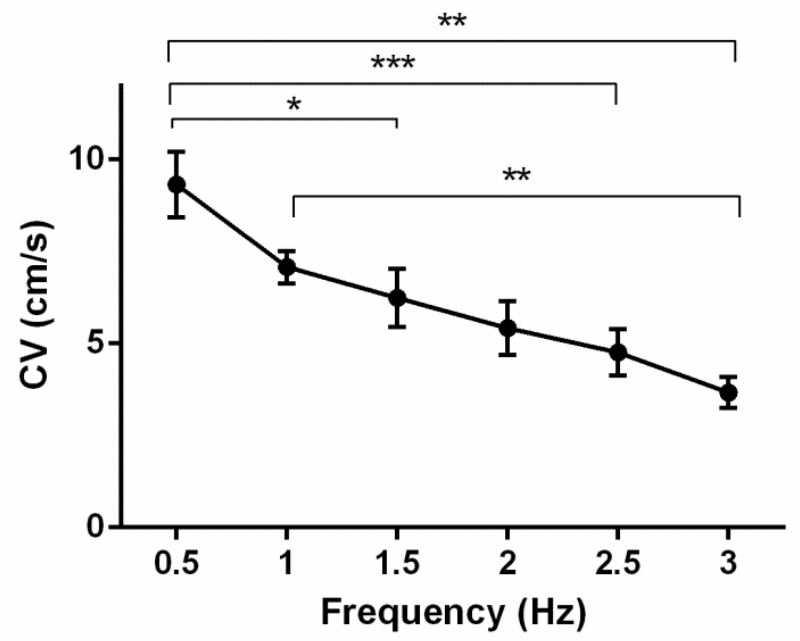

Baseline

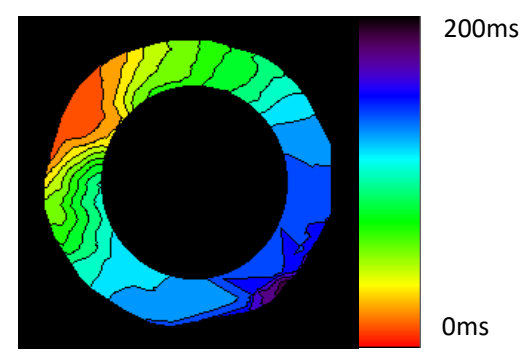

Baseline

C

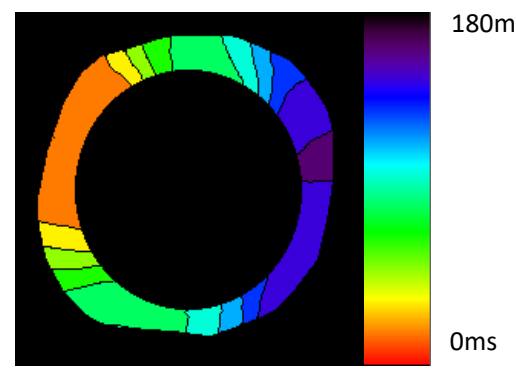

Lidocaine - $100 \mu \mathrm{M}$

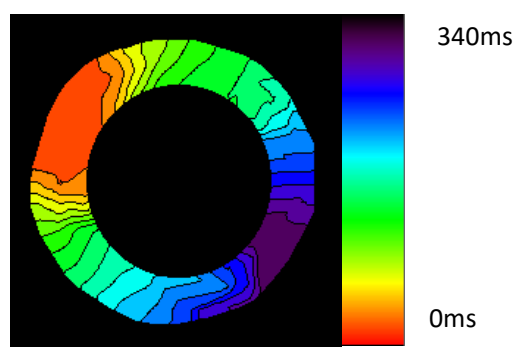

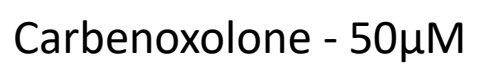

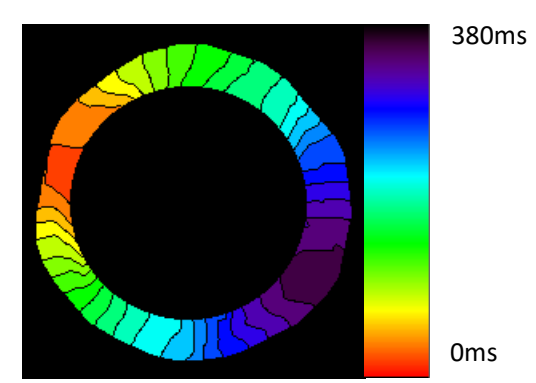

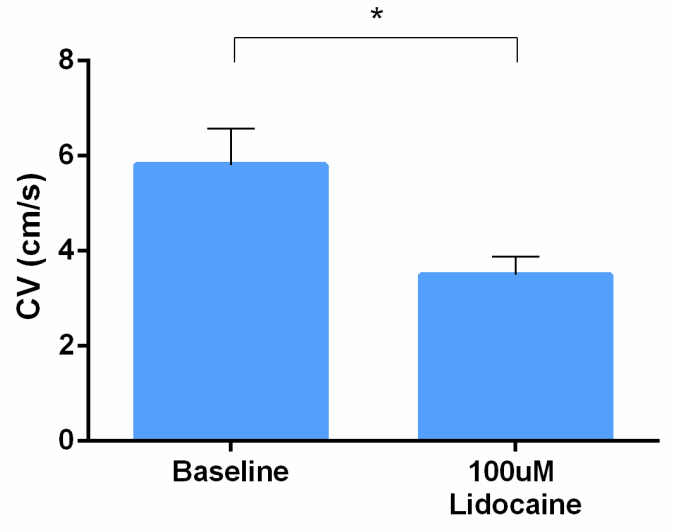

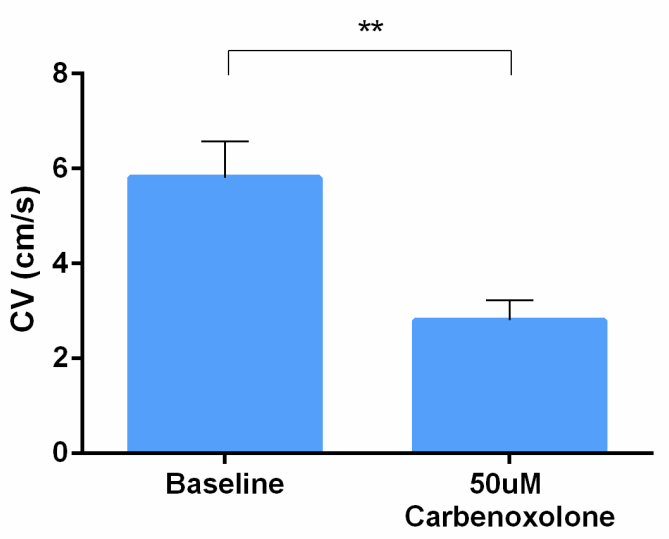


D
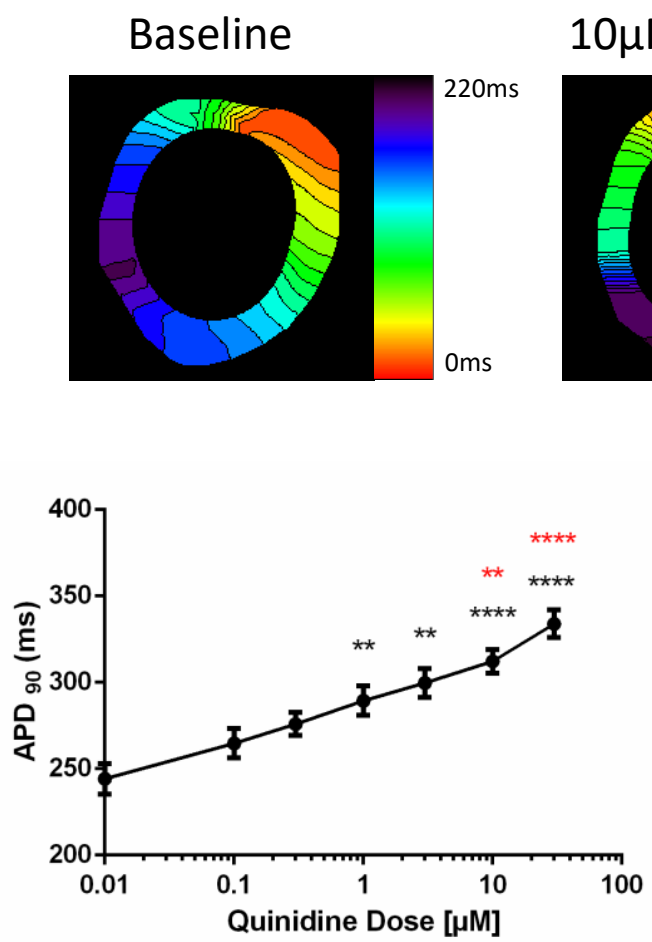

E
LQTS:

Baseline

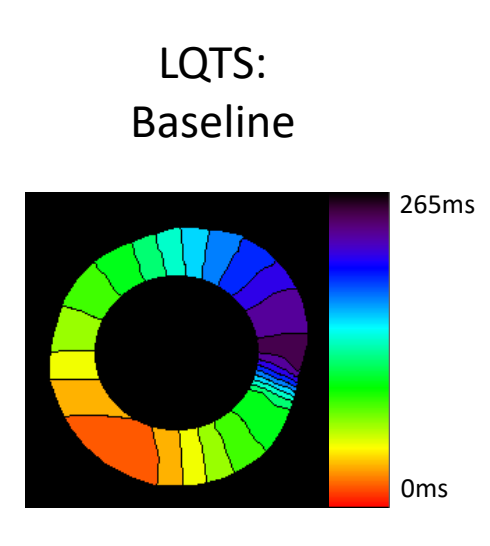

E
$10 \mu \mathrm{M}$ quinidine

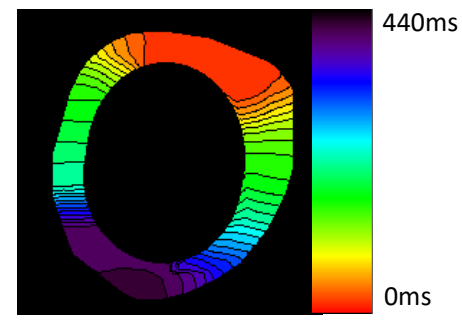

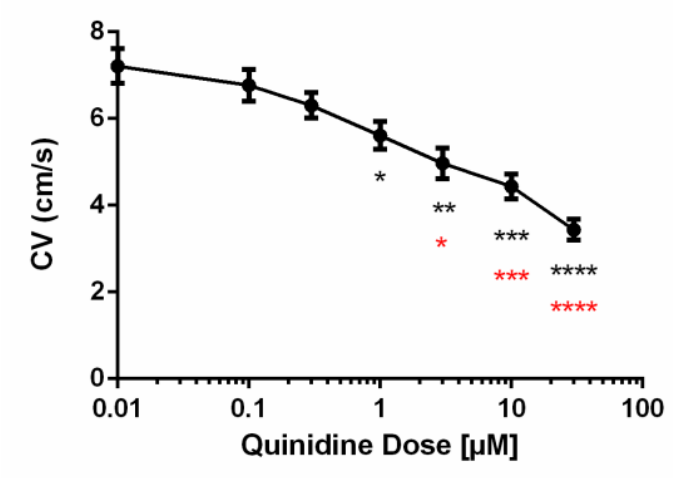

F

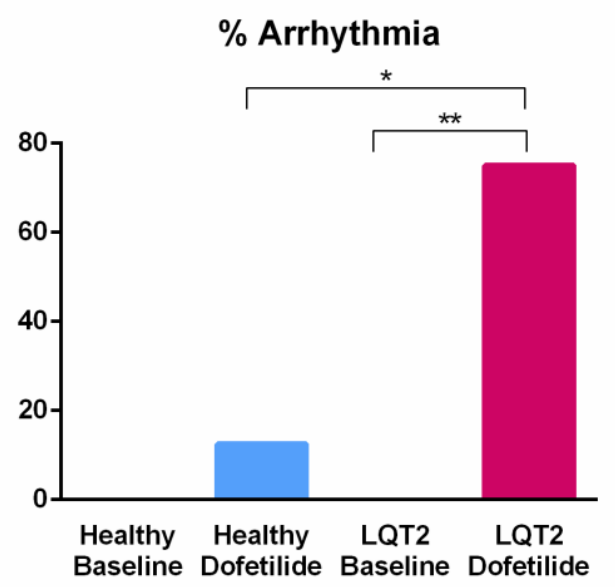

